\title{
Joint Clustering and Discriminative Feature Alignment for Unsupervised Domain Adaptation
}

\author{
Wanxia Deng, Qing Liao, Lingjun Zhao, Deke Guo, Gangyao Kuang, Dewen Hu, and Li Liu
}

\begin{abstract}
Unsupervised Domain Adaptation (UDA) aims to learn a classifier for the unlabeled target domain by leveraging knowledge from a labeled source domain with a different but related distribution. Many existing approaches typically learn a domain-invariant representation space by directly matching the marginal distributions of the two domains. However, they ignore exploring the underlying discriminative features of the target data and align the cross-domain discriminative features, which may lead to suboptimal performance. To tackle these two issues simultaneously, this paper presents a Joint Clustering and Discriminative Feature Alignment (JCDFA) approach for UDA, which is capable of naturally unifying the mining of discriminative features and the alignment of class-discriminative features into one single framework. Specifically, in order to mine the intrinsic discriminative information of the unlabeled target data, JCDFA jointly learns a shared encoding representation for two tasks: supervised classification of labeled source data, and discriminative clustering of unlabeled target data, where the classification of the source domain can guide the clustering learning of the target domain to locate the object category. We then conduct the cross-domain discriminative feature alignment by separately optimizing two new metrics: 1) an extended supervised contrastive learning, i.e., semi-supervised contrastive learning 2) an extended Maximum Mean Discrepancy (MMD), i.e., conditional MMD, explicitly minimizing the intra-class dispersion and maximizing the inter-class compactness. When these two procedures, i.e., discriminative features mining and alignment are integrated into one framework, they tend to benefit from each other to enhance the final performance from a cooperative learning perspective. Experiments are conducted on four real-world benchmarks (e.g., Office-31, ImageCLEFDA, Office-Home and VisDA-C). All the results demonstrate that our JCDFA can obtain remarkable margins over stateof-the-art domain adaptation methods. Comprehensive ablation studies also verify the importance of each key component of our proposed algorithm and the effectiveness of combining two learning strategies into a framework.
\end{abstract}

Index Terms-Domain Adaptation, Deep Learning, Transfer Learning, Unsupervised Learning, Semisupervised Learning

This work was partially supported by the Academy of Finland under grant 331883, and the National Natural Science Foundation of China under Grant 61872379, 62022091 and 71701205 .

Li Liu is with the College of System Engineering, National University of Defense Technology, Changsha, Hunan, China, and is also with the Center for Machine Vision and Signal analysis, University of Oulu, Oulu, Finland Li Liu is the corresponding author. (email: li.liu@oulu.fi)

Wanxia Deng, Lingjun Zhao and Gangyao Kuang are with College of Electronic Science, National University of Defense technology, Changsha, Hunan, China. (email: wanxiadeng@163.com, nudtzlj@163.com, kuanggangyao@nudt.edu.cn)

Deke Guo is with the College of System Engineering, National University of Defense Technology, Changsha, Hunan, China. (email: guodeke@gmail.com)

Dewen $\mathrm{Hu}$ is with the College of Intelligent Science, National University of Defense Technology, Changsha, Hunan, China. (email: dwhu@nudt.edu.cn)

Qing Liao is with the Department of Computer Science and Technology, Harbin Institute of Technology, Shenzhen, China. (email: liaoqing@hit.edu.cn)

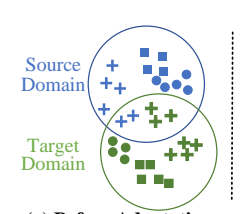

(a) Before Adaptation

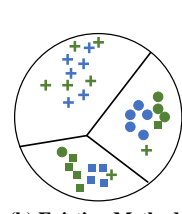

(b) Existing Methods

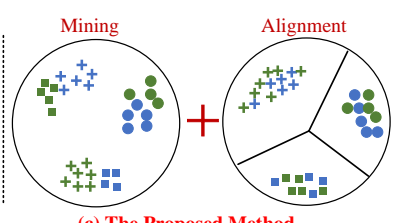

(c) The Proposed Method
Fig. 1. The comparison of existing methods and the proposed method. (a) The before adaptation for source and target domains. (b) Existing methods directly align the marginal distributions while ignoring the intrinsic discriminative information and the class-level structures cannot perform well in these cases. (c) The proposed method JCDFA employs the clustering learning of the target domain to mine the intrinsic discriminative features and aligns the class-level discriminative features corporately. Meanwhile, the clustering is guided by the supervised classification of the source domain.

\section{INTRODUCTION}

Deep neural networks has brought great success in various computer vision tasks, including image classification [1], object detection [2], image segmentation [3], [4], and face recognition [5], among many others. Progress in computer vision is significant, but what we are witnessing is a supervised learning revolution. Undoubtedly, fully supervised learning heavily depends on large amounts of labeled samples that are labor-intensive and time-consuming to obtain. Also, there are many scenarios where labeling data is extremely expensive or even impossible due to privacy or other issues. Nowadays, unlabeled data requires lower cost to collect, but there lack of effective ways to exploit them.

Domain Adaptation (DA), a subfield of transfer learning, has emerged as one appealing paradigm to address such problems, and numerous methods have been proposed [6], [7], [8], [9]. The objective of DA is to leverage labeled data from one or more similar domains (named source domain) to improve the learning of the interested domain (named target domain) that has a distribution different from but related to the source distribution [10], i.e., the domain shift problem [11], [12]. Therefore, in the field of DA, how to address the domain shift between the source and target becomes critical. In this paper, we tackle the problem of Unsupervised DA (UDA), where the algorithm has access to labeled samples from a source domain and unlabeled data from a target domain.

One popular research thread for UDA is to learn domaininvariant features [13], [14], [15], [16], [17]. Mainstream approaches either explicitly extract the domain-invariant features via minimizing the domain discrepancy which is measured by Maximum Mean Discrepancy (MMD) [18], [19], [20], Joint MMD (JMMD) [21], weighted MMD [22], [23] CORrelation 
(CORAL) alignment [24] and Central Moment Discrepancy (CMD) [25], or implicitly learn the domain-invariant features via adversarial learning which is implemented by introducing domain discriminator [26], [27], [28], conditional domain discriminator [29], adversarial mechanism of task classifiers [30], [31]. Despite their general efficacy for UDA, their performance may still be constrained by two bottlenecks. First, these methods overlook the underlying discriminative representations of the unlabeled target domain. Although unlabeled, the intrinsic structure of target data manifests distinguishability. Exploring such discriminative information can contribute to accelerating the domain-invariant features learning, reducing the class-level distribution discrepancy between the two domains. Second, many existing methods assume that a classifier trained with source data performs well on the target domain; thus, they only align the marginal distributions of the two domains, and ignore the difference of class-level features, e.g., conditional distributions. Unfortunately, this may result in misclassification, as shown in Figure 1 (b). For instance, it is possible that, even though the marginal distribution of the source and target domains are perfectly aligned, and yet a square sample in the source domain maps to a circle sample in the target domain. The discrimination of the learned features is therefore also of great importance for identifying the unlabeled target data.

To tackle the two challenges mentioned above simultaneously, we propose a new approach named Joint Clustering and Discriminative Feature Alignment (JCDFA) where we naturally unify the discriminative information exploration of the target domain and cross-domain adaptation with classdistinguishable features preserved into one framework. Figure 1 (c) shows our motivation about combining clustering learning and discriminative feature alignment. The left picture of the Figure 1 (c) depicts that we firstly promote the classdistinguishable structure via learning the class boundaries in the source domain and the clusters boundaries in the target domain, and then apply the discriminative features to conduct class-wise alignment as shown in the right picture of the Figure 1 (c).

To be specific, firstly, we intend to learn the discriminative feature of the target domain. There are two mainstream approaches, e.g., reconstruction and clustering, to mine the intrinsic structure of unlabeled data. One is the reconstruction of unlabeled data using an autoencoder. One representation work is DRCN [32] which extracts domain-invariant features by learning the shared encoding via the supervised classification of the source domain and the unsupervised reconstruction of the target domain. In this approach, however, the reconstruction attempts to capture all of the information contained in the image; thus, the features learned are not discriminative which may lead to suboptimal adaptation performance. Moreover, the reconstruction introduces a decoder branch, which will further increase the training complexity. We therefore choose the clustering of the unlabeled data to mine the discriminative information. We learn discriminative features via the supervised classification of the source domain and the unsupervised clustering of the target domain employing a shared encoding. The shared encoding is trained by the supervised classification loss of the source domain and the unsupervised clustering loss of the target domain. The clustering loss is defined as the Kullback-Leibler (KL) divergence between the model's predictive label distribution and an introduced auxiliary one [33], [34]. With the supervised classification guidance using the shared encoder, the clustering learning can reveal the classlevel discriminative information of the target domain. By this means, the learned representation can guarantee categorical distinguishability while obtaining a certain domain-invariance for these two domains.

The discriminative features have been achieved for each domain. However, ensuring that the corresponding categories of the two domains are correctly matched is the second challenge mentioned above. In this regard, we conduct the class-wise alignment via encouraging the minimum distance of data pair in the same class and the maximum distance of data pair sharing the different labels across domains in two metric ways, respectively.

The first one is modifying the recently proposed Supervised Contrastive Learning (SupCon) [35], which builds on the contrastive self-supervised literature by leveraging label information. Accordingly, we write the proposed method as JCDFA-C. SupCon [35] defines a supervised contrastive loss constraining that normalized embeddings from the same class are pulled closer together than embeddings from different classes. Inspired by this, we extend the supervised contrastive loss to semi-supervised contrastive loss for UDA. In detail, the category labels of unlabeled samples in the target domain are first predicted and adopted as pseudo-labels through the spherical clustering during the training process. We then incorporate class-discriminative feature alignment by encouraging that samples belonging to the same class are pulled together across domains. To strengthen the alignment of the discriminative features, we further modify the proposed semi-supervised contrastive loss and add a new loss item that can ensure the inter-class maximum distance by pushing apart samples from different classes. Following the strategies, we explicitly minimize intra-class distance and maximize inter-class distance across domains via the proposed semisupervised contrastive loss, which can lead to the alignment of class-level distributions.

The second one is explicitly minimizing the Maximum Mean Discrepancy (MMD) [36] distances of conditional distributions across domains, and we rewrite the proposed method as JCDFA-M. Similarly, we use pseudo-labels generated by spherical clustering during the training process. We then introduce the class-specific information into the original MMD for exploiting the conditional distribution adaptation. The intra-class MMD distance and inter-class MMD distance are minimized and maximized, respectively, which leads the learned representation to adapt the conditional distribution shift.

Our overall goal is to integrate the learning of discriminative features and the adaptation of class-distinguishable features into a unified framework. We couple the mining of the discriminative features and the adaptation of the classdistinguishable features in a mutually beneficial manner. The target pseudo-labels can be refined based on the clustering 
updating and the adaptation of class-level distributions. Moreover, the exploration of the class-discriminatory information can aid in keeping different classes of each domain far away from each other and the identical classes of each domain closer to each other, while the learning strategy significantly diminishes the cross-domain marginal distribution discrepancy via the shared encoding. The good class-separability features will also facilitate the class-level distribution adaptation. The contributions of our work can be summarized as follows:

(1) We propose a novel approach named JCDFA to address two critical bottlenecks for UDA simultaneously. First, the learned domain-invariant representations can be equipped with category-discriminative knowledge via the joint learning of supervised classification of the source domain and unsupervised clustering of the target domain. Second, the classlevel distribution can be adapted by keeping the identical classes closer to each other and different classes far away from each other across domains via two metric ways, i.e., an extended supervised contrastive learning and an extended MMD distance. Accordingly, for the sake of clarity, the proposed method JCDFA is renamed as JCDFA-C and JCDFA$\mathrm{M}$, respectively.

(2) The proposed JCDFA ${ }^{1}$ explicitly unifies the learning of features and the adaptation of class-distinguishable features into a unified framework. JCDFA can fully exploit the discriminative information of both domains and effectively minimize the class-level distribution divergences between these domains simultaneously, thereby facilitate the learning process and boost the classification performance.

(3) For JCDFA-C, we extend the recently proposed SupCon to semi-supervised contrastive learning for UDA. The proposed semi-supervised contrastive loss further introduces the maximum of the inter-class distance, which can strengthen better features discriminability.

(4) Comprehensive experiments on the Office-31, ImageCLEF-DA, Office-Home and VisDA-C datasets are conducted to demonstrate that the proposed method outperforms existing methods by a large margin. Furthermore, a number of mutually beneficial ablation studies are conducted to prove the exploration of the discriminative information and class-level features alignment can benefit from each other.

\section{RELATED WORK}

This section reviews mainstream approaches in UDA and focuses on some approaches that are related to our work. Besides, considering that our work draws on the contrastive learning, we will briefly review this topic.

\section{A. Mainstream Approaches in UDA}

Metric discrepancy-based methods One classical approach to UDA involves directly minimizing the distribution discrepancy of domains via the metric paradigm. Some representative metric methods include Maximum Mean Discrepancy (MMD) [18], [21], CORrelation (CORAL) alignment

\footnotetext{
${ }^{1}$ In our following presentation, the JCDFA refers to JCDFA-C and JCDFA$\mathrm{M}$ collectively for simplicity.
}

[24] and Central Moment Discrepancy (CMD) [25]. In [18] and [21], the distribution discrepancy is minimized via a variant of MMD, e.g. Multi-Kernel MMD (MK-MMD) and Joint Maximum Mean Discrepancy (JMMD), respectively. The authors of [22] exploit the class prior probability via a weighted MMD model that introduces class-specific auxiliary weights into the original MMD. Moreover, D-CORAL [37] applies CORAL to align the correlations of layer activations in deep neural networks. Central Moment Discrepancy (CMD) [25] was also developed to match the higher-order central moments of probability distributions.

Adversarial learning-based methods Another popular branch of UDA is based on the adversarial learning of domains, inspired by the Generative Adversarial Network (GAN) [38]. RevGrad [26], DANN [27], Adversarial Discriminative Domain Adaptation (ADDA) [28] and Conditional Domain Adversarial Network (CDAN) [29] all utilize a domain discriminator to represent the domain discrepancy. The domain discriminator is confused in a two-player minimax game. The Wasserstein Distance Guided Representation Learning (WDGRL) [39] and Re-weighted Adversarial Adaptation Network (RAAN) [40] estimate the distribution distance between the source and target samples in a domain critic network and optimize the feature extractor network to minimize the estimated distance in an adversarial manner. Unlike the form of domain adversarial learning discussed above, Maximum Classifier Discrepancy (MCD) [30] and Sliced Wasserstein discrepancy (SWD) [31] define a new adversarial standard in developing generic DA frameworks. The MCD and SWD utilize task-specific classifiers as discriminators and align the target and source distributions using the adversarial learning or the Wasserstein metric of two task-specific classifiers, respectively. Domainsymmetric Networks (SymNets) in [41] presents a novel adversarial learning objective, the key design of which is based on a two-level domain confusion scheme. Transferable Adversarial Training (TAT) [42] generates transferable examples to fill the gap between the source and target domains, and further adversarially trains the deep classifiers to make consistent predictions over the transferable examples. $\mathrm{Gu}$ et al. [43] proposes a novel adversarial DA approach named Robust Spherical Domain Adaption (RSDA), in which spherical classifier and spherical domain discriminator are defined for label prediction and discriminating domain labels, respectively. Besides, RSDA defines a novel robust pseudo-label loss in spherical feature space for utilizing target pseudo-labels more robustly. On the basis of domain alignment using adversarial learning, Dynamic Weighted Learning (DWL) [44] proposes dynamically weighted learning between domain alignment and class discriminability by weighting the samples according to the imbalance degree of the sample number of the two domains.

Clustering learning-based methods The proper incorporation of the class-conditional structure via clustering has been shown to be beneficial in various tasks. Early work of this kind [45] makes the discriminative clustering assumption that the data in both the source and target domains are tightly clustered and that clusters correspond to class boundaries. 
However, one of the limitations of [45] is that it uses a simple linear transformation to learn the feature space, which is less able to extract high-order features from original data effectively. The Cluster Alignment with a Teacher (CAT) [46] applies a Fisher-like criterion-based deep clustering loss [47] and leverages an implicit ensembling teacher model to uncover the class-conditional structure of domains. It then aligns the corresponding clusters across domains using the first-order statistics of distributions. However, CAT applies target clustering only as an incremental technique to improve explicit feature alignment. Moreover, the alignment of the corresponding cluster distribution is learned only by the intraclass distance, which is insufficient to explore the conditional distribution discrepancy. The work in [48] employs pair-wise KL-divergence to define the clustering loss. The Structurally Regularized Deep Clustering (SRDC) [49] defines the target discrimination using the clustering of intermediate network features [33], [34]. Although the two methods proposed in [50], [48] have achieved much better performance, they ignore the fact that the exploration of discriminative features cannot guarantee the alignment of the corresponding categorical features of two domains; accordingly, there is a need to adapt the condition distribution.

Class-level alignment-based methods Class-level transfer aims to decrease the discrepancy of the conditional distribution. Moving Semantic Transfer Network (MSTN) [51] aligns the labeled source centroid and the pseudo-labeled target centroid to learn domain-invariant semantic representations. SimNet [52] and Transferable Prototypical Networks (TPN) [53] learn categorical prototype representations by computing the similarity between the prototype representations of each category. CWDAN [23] employs the MMD metric to minimize the intra-class distance across domains, while the Contrastive Adaptation Network (CAN) [20] explicitly models the intraclass domain discrepancy and the inter-class domain discrepancy based on the MMD metric. MADA [54] trains multiple class-wise domain discriminators to capture multi-mode structures, thereby enabling the fine-grained alignment of different data distributions. Similarly, GSDA [50] implements hierarchical domain alignments including class-wise, groupwise and global alignment. Although this type of method significantly boosts performance by exploring the class-wise adaptation, there is also a drawback that many class-level alignment methods incorporate target pseudo labels, most of which depend only on the source classifier. It should be noted here that pseudo-labeling is a double-edged sword, as wrongly predicted target labels will mislead the class-wise distribution alignment, resulting in performance degradation. It is therefore crucial to guarantee the accuracy of targeted pseudo-labels in order to learn proper domain-invariant discriminative features. To refine the pseudo labels, Chen et al.[55] proposes deep credible metric learning (DCML) method for crossdomain person re-identification. DCML progressively mines credible samples using the k-Nearest Neighbor similarity for density evaluation and the prototype similarity for centrality evaluation. Different with DCML [55], our proposed method incorporates clustering learning into the class-level alignment, as this approach can help find the class-wise boundary that promotes pseudo-label prediction. The clustering learning procedure also further facilitates the class-wise alignment.

\section{B. Contrastive visual representation learning}

Contrastive learning has been used for self-supervised visual representation learning [56], [57], [58] and has lead to great empirical success, where a contrastive loss is adopted to learn instance discriminative representations by regarding each unlabeled sample as a distinct class and contrasting positive pairs against negative pairs. Although instance-level contrast loss can be applied to train embedding which can be well extended to downstream tasks, it cannot show great promise for domain adaptive object recognition tasks that need to measure the class-level affinity. Different from the self-supervised contrastive learning, the recently proposed Supervised Contrastive Learning (SupCon) [35] builds on the different basis of the contrastive learning, which has changed from "whether it comes from the same picture" to "whether it belongs to the same category". SupCon pursues to develop a contrastive loss function that allows the effective merging of labeled data while retaining the favorable characteristics of the contrastive loss which is crucial for the success of selfsupervised representation learning. Based on the simplicity, stability and learned feature discriminability, we modify this SupCon loss to be suitable for UDA and extend the supervised contrastive learning to semi-supervised contrastive learning, while simultaneously preserving properties important to the self-supervised approach. Recently, Ge et al. [59] proposes a novel self-paced contrastive learning framework with hybrid memory for cross-domain object Re-ID. Different from ours, the work [59] applies the three components: source-domain classes, target-domain clusters and un-clustered instances as the supervisory signal of the contrastive learning, while our proposed JCDFA-C introduces the category supervisory signal into the contrastive learning to conduct the class-level features alignment.

\section{Proposed Methodology}

In this section, we first present the UDA problem formulation, then introduce the proposed Joint Clustering and Discriminative Feature Alignment (JCDFA) approach, with a focus on the exploration of discriminative features and the adaptation of the class-level discriminative feature via semisupervised contrastive learning and extended MMD distance. Accordingly, the JCDFA-C and JCDFA-M will be illustrated. The framework of JCDFA-C is depicted in Figure 2. The proposed method includes the two stages: 1) computing the pseudo labels and clustering centroids of unlabeled target domain; 2) the mining and alignment of discriminative features. The clustering centroids are utilized to mine the discriminative features. The pseudo labels are applied to align discriminative features. And the model learning via the mining and alignment of discriminative features can further improve the accuracy of pseudo labels and clustering centroids. These two stages work iteratively and promote each other. For simplicity, Figure 3 only presents the specific part of the JCDFA-M not repeating the same part with JCDFA-C. 

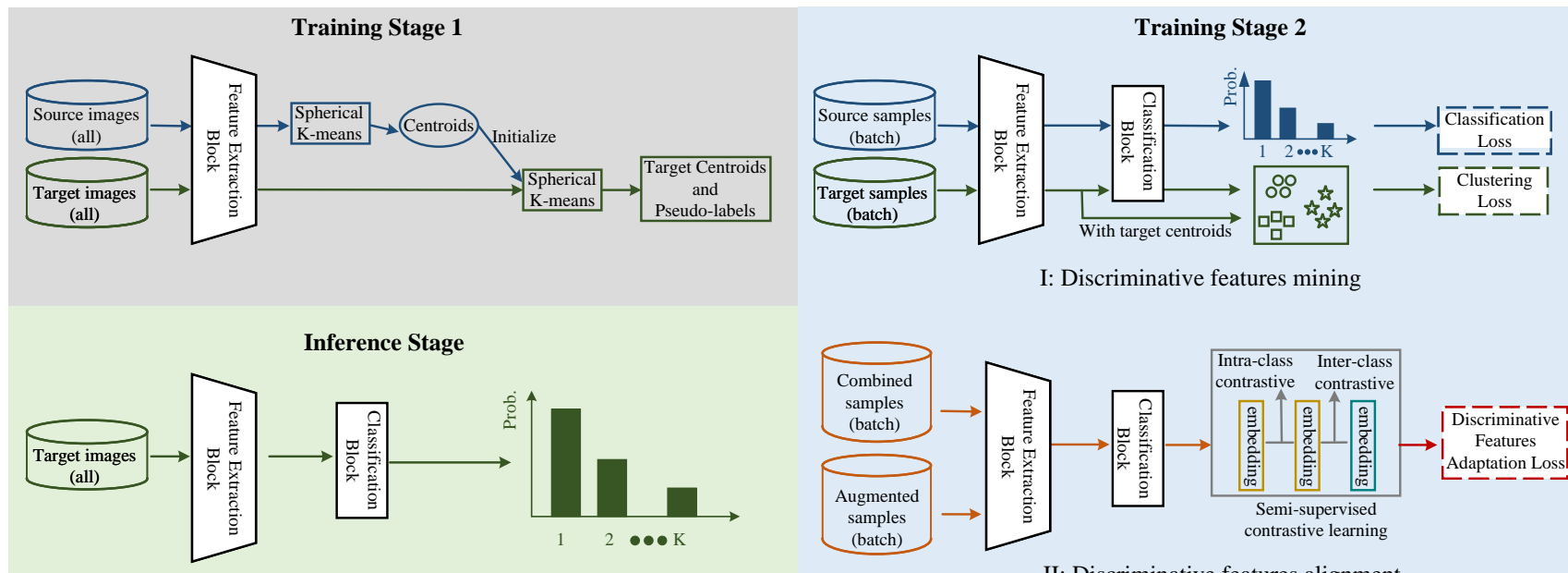

I: Discriminative features mining

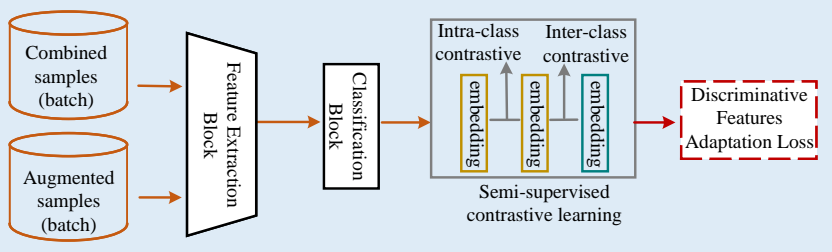

II: Discriminative features alignment

Fig. 2. The training and inference stages of the proposed JCDFA-C. The training of JCDFA-C includes two stages, and they work iteratively. At the first training stage, the whole source images and target images are applied to calculate the target centroid and its pseudo labels. The second stage includes two procedures, i.e., discriminative features mining and alignment, and they work cooperatively. In the procedure I, a batch of images are sampled from the two domains, respectively. The standard supervised classification loss of the sampled source domain and the unsupervised clustering loss of the sampled target domain are employed to mine the domain-invariant discriminative features. In the procedure II, the sampled source and target domain are mixed up and further augmented. The semi-supervised contrastive loss is employed to adapt the discriminative representations across domains by making constrative distances of the intra-class embedding minimize, and those of the inter-class embedding maximize. Notably, the colors of blocks and lines represent their corresponding data flows.

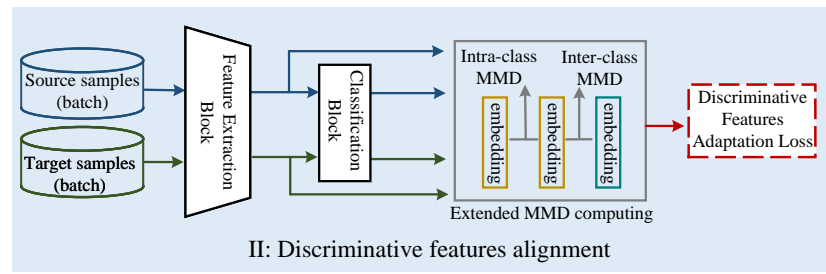

Fig. 3. The discriminative features alignment procedure of the proposed JCDFA-M. The sampled source and target domains are applied to align the class-level distributions via the extended MMD distance, where the MMD distance of the embedding belonging to the same class is minimized, and its of the embedding from different classes is minimized. Here, JCDFA-M conducts discriminative features adaptation employing multi-layers embedding. Thus, the embedding can be from the feature extraction block, and also can be obtained from the classification block. Notably, the colors of blocks and lines represent their corresponding data flows.

\section{A. The UDA Problem Formulation}

We focus on the problem of UDA in image classification, where we consider two different domains defined with different but related probability distributions $p(\boldsymbol{x}, y)$ over the input-label space pair $X \times Y$. In more detail, the domain of interest is dubbed the target domain with distribution $p_{t}(\boldsymbol{x}, y)$ while the available domain with labeled data is called the source domain and has distribution $p_{s}(\boldsymbol{x}, y)$. Let $|Y|=C$, and $y \in 1,2, \ldots, C$. The goal of the UDA task is to predict the labels of samples drawn from a target domain as accurately as possible, given $N_{s}$ labeled samples $X^{s}=\left\{\boldsymbol{x}_{i}^{s}\right\}_{i=1}^{N_{s}}$, with the annotations $Y^{s}=\left\{y_{i}^{s}\right\}_{i=1}^{N_{s}}$ drawn from a source domain and $N_{t}$ unlabeled samples $X^{t}=\left\{\boldsymbol{x}_{i}^{t}\right\}_{i=1}^{N_{t}}$ sampled from the target domain. Formally, we define the feature extractor as $f$ with parameters $\boldsymbol{\theta}$ and the embedding classifier as $g$ with parameters $\phi$. We denote the whole network as $h=f \circ g$.

\section{B. Exploration of Discriminative Features}

In this section, we will introduce how the discriminative information is mined for the two domains. The supervised classification of the source domain and the unsupervised clustering of the target domain are learned cooperatively via shared encoding in order to extract the discriminative features. From a technical perspective, we define the supervised classification loss of the source domain as:

$$
\mathcal{L}_{c l s}(\boldsymbol{\theta}, \boldsymbol{\phi})=\frac{1}{N_{s}} \sum_{i=1}^{N_{s}} \ell_{c e}\left(h\left(\boldsymbol{x}_{i}^{s} ; \boldsymbol{\theta}, \boldsymbol{\phi}\right), y_{i}^{s}\right),
$$

where $\ell_{c e}$ denotes the cross-entropy loss. Meanwhile, we consider the clustering learning of the target domain $X^{t}=$ $\left\{\boldsymbol{x}_{i}^{t}\right\}_{i=1}^{N_{t}}$ which is clustered into $C$ clusters in the output probability space. We define the prediction of the network, following a multinomial logistic regression operation (i.e., softmax), as a vector $\left\{p_{i}^{t}\right\}_{i=1}^{N_{t}}$ which we abbreviate to $P^{t}$. The element $p_{i c}^{t}$ of $P^{t}$ means the probability of assigning $\boldsymbol{x}_{i}^{t}$ to the class $c$. Similar to [34], we first introduce an auxiliary target variable $Q^{t}$. The clustering objective function can thus be defined as:

$$
\begin{aligned}
\mathcal{L}_{c l u}^{\prime}(\boldsymbol{\theta}, \boldsymbol{\phi}) & =K L\left(Q^{t} \| P^{t}\right) \\
& =\frac{1}{N_{t}} \sum_{i=1}^{N_{t}} \sum_{c=1}^{C} q_{i c}^{t} \log \frac{q_{i c}^{t}}{p_{i c}^{t}}+q_{i c}^{t} \log \varrho_{k}^{t},
\end{aligned}
$$

where the first term denotes the KL divergence between the model prediction probability $P^{t}$ and the auxiliary target variable $Q^{t}$. The second term is used to enforce the balanced assignments, where $\varrho_{k}^{t}=\frac{1}{N_{t}} \sum_{i=1}^{N_{t}} q_{i c}^{t}$. $q_{i c}^{t}$ denotes the element of $Q^{t}$, which is defined as follows:

$$
q_{i c}^{t}=\frac{p_{i c}^{t} /\left(\sum_{j=1}^{N_{t}} p_{j c}^{t}\right)^{\frac{1}{2}}}{\sum_{c^{\prime}=1}^{C} p_{i c^{\prime}}^{t} /\left(\sum_{j=1}^{N_{t}} p_{j c^{\prime}}^{t}\right)^{\frac{1}{2}}} .
$$


To strengthen the discriminative ability of the learned feature, we further introduce the clustering learning in the latent feature space $Z^{t}$ which is defined in the last layer output of the feature extractor, i.e., $\boldsymbol{z}_{i}^{t}=f\left(\boldsymbol{x}_{i}^{t} ; \boldsymbol{\theta}\right) \in Z^{t}$. Similarly, we partition the data in the latent feature space $Z^{t}$ into $C$ clusters, each of which is represented by a centroid $\boldsymbol{\mu}_{c}^{t}, \quad c=1, \ldots, C$, where $\boldsymbol{\mu}_{c}^{t} \in Z^{t}$ and the cluster centroid is learnable. The target cluster centroid $\boldsymbol{\mu}_{c}^{t}$ is first initialized using the source cluster centroid $\boldsymbol{\mu}_{c}^{s}$, i.e., $\boldsymbol{\mu}_{c}^{s} \rightarrow \boldsymbol{\mu}_{c}^{t}$, where $\boldsymbol{\mu}_{c}^{s}=\sum_{i=1}^{N_{s}} \mathbf{1}_{y_{i}^{s}=c} \frac{f\left(\boldsymbol{x}_{i}^{s}\right)}{\left\|f\left(\boldsymbol{x}_{i}^{s}\right)\right\|}, \mathbf{1}_{\hat{y}_{i}^{s}=c}=\left\{\begin{array}{ll}1 & \text { if } \hat{y}_{i}^{s}=c \\ 0 & \text { otherwise }\end{array}\right.$. Then we cluster the target samples using spherical K-means to obtain their pseudo labels $\left\{\hat{y}_{i}^{t}\right\}_{i}^{N_{t}}$. We compute the cosine distance $\operatorname{dist}\left(\boldsymbol{z}_{i}^{t}, \boldsymbol{\mu}_{c}^{t}\right)$ between the target embedding $\boldsymbol{z}_{i}^{t}$ and the target cluster center $\boldsymbol{\mu}_{c}^{t}$, where $\operatorname{dist}\left(\boldsymbol{z}_{i}^{t}, \boldsymbol{\mu}_{c}^{t}\right)=\frac{1}{2}(1-$ $\left.\frac{<z_{i}^{t}, \boldsymbol{\mu}_{c}^{t}>}{\left\|z_{i}^{t}\right\|\left\|\boldsymbol{\mu}_{c}^{t}\right\|}\right)$. According to the $\operatorname{argmin}_{c} \operatorname{dist}\left(\boldsymbol{z}_{i}^{t}, \boldsymbol{\mu}_{c}^{t}\right)$, we can get the pseudo label $\hat{y}_{i}^{t}$ and further update the target cluster centroid using $\boldsymbol{\mu}_{c}^{t}=\sum_{i=1}^{N_{t}} \mathbf{1}_{\hat{y}_{i}^{t}=c} \frac{f\left(\boldsymbol{x}_{i}^{t}\right)}{\left\|f\left(\boldsymbol{x}_{i}^{t}\right)\right\|}$. Following [60], we use the Student's t-distribution as a kernel to measure the probability assigning embedded point $\boldsymbol{z}_{i}^{t}$ to centroid $\boldsymbol{\mu}_{c}^{t}$, as follows:

$$
\tilde{p}_{i c}^{t}=\frac{\exp \left(\left(1+\left\|\boldsymbol{z}_{i}^{t}-\boldsymbol{\mu}_{c}^{t}\right\|^{2}\right)\right)^{-1}}{\sum_{c^{\prime}=1}^{C} \exp \left(\left(1+\left\|\boldsymbol{z}_{i}^{t}-\boldsymbol{\mu}_{c^{\prime}}^{t}\right\|^{2}\right)\right)^{-1}},
$$

where $\tilde{p}_{i c}^{t}$ represents the probability of soft cluster assignments based on instance-to-centroid distances in the latent feature space $Z^{t}$. We collectively write $\tilde{p}_{i c}^{t}$ as $\tilde{P}_{\tilde{\tilde{Q}}}^{t}$. Following [33], we introduce the auxiliary distribution $\tilde{Q}^{t}$ to conduct the clustering learning. Similar to Equation 3, the element of $\tilde{Q}^{t}$ is defined as follows:

$$
\tilde{q}_{i c}^{t}=\frac{\tilde{p}_{i c}^{t} /\left(\sum_{j=1}^{N_{t}} \tilde{p}_{j c}^{t}\right)^{\frac{1}{2}}}{\sum_{c^{\prime}=1}^{C} \tilde{p}_{i c^{\prime}}^{t} /\left(\sum_{j=1}^{N_{t}} \tilde{p}_{j c^{\prime}}^{t}\right)^{\frac{1}{2}}} .
$$

The clustering loss of the latent feature layer can be defined as:

$$
\begin{aligned}
\mathcal{L}_{c l u}^{\prime \prime}(\boldsymbol{\theta}) & =K L\left(\tilde{Q}^{t} \| \tilde{P}^{t}\right) \\
& =\frac{1}{N_{t}} \sum_{i=1}^{N_{t}} \sum_{c=1}^{C} \tilde{q}_{i c}^{t} \log \frac{\tilde{q}_{i c}^{t}}{\tilde{p}_{i c}^{t}}+\tilde{q}_{i c}^{t} \log \tilde{\varrho}_{k}^{t},
\end{aligned}
$$

where $\tilde{\varrho}_{k}^{t}=\frac{1}{N_{t}} \sum_{i=1}^{N_{t}} \tilde{q}_{i c}^{t}$. By combining Equations 2 and 6, we can obtain the overall clustering loss:

$$
\mathcal{L}_{c l u}(\boldsymbol{\theta}, \boldsymbol{\phi})=\mathcal{L}_{c l u}^{\prime}(\boldsymbol{\theta}, \boldsymbol{\phi})+\mathcal{L}_{c l u}^{\prime \prime}(\boldsymbol{\theta}) .
$$

By training the classification loss (i.e., Equation 1) and the clustering loss (i.e., Equation 7) together, the parameters of the model and the clustering centroids can be gradually updated. In this way, we can obtain domain-invariant categorydiscriminative representations.

\section{Adaptation of Discriminative Features}

Minimizing Equations 1 and 7 simultaneously via the shared encoding enables us to obtain the discriminative, yet domaininvariant features. However, the discriminative information obtained is insufficient to guarantee the classification accuracy for the target domain. $\overline{\text { Algorithm } 1 \text { Joint Clustering and Discriminative Feature Alignment }}$

\section{Input:}

source data: $X^{s}=\left\{\boldsymbol{x}_{i}^{s}\right\}_{i=1}^{N_{s}}$, with the annotations $Y^{s}=$ $\left\{y_{i}^{s}\right\}_{i=1}^{N_{s}}$.

target data: $X^{t}=\left\{\boldsymbol{x}_{i}^{t}\right\}_{i=1}^{N_{t}}$.

Output: the predicted label $y^{t}$ for target domain unlabeled data.

Initialization: Initialize feature extractor network $f$ and classifier network $g$, and set the hyperparameters.

While not converge and epoch $<$ max_epoch do

1) Forward $X^{s}$ and compute the $C$ cluster centers $\boldsymbol{\mu}_{c}^{s}$.

2) Initialize $\boldsymbol{\mu}_{c}^{s} \rightarrow \boldsymbol{\mu}_{c}^{t}$.

3) Forward $X^{t}$, cluster the target samples according to the initialized target clusters $\boldsymbol{\mu}_{c}^{t}$ and the embedding $Z^{t}$, and assign the pseudo label for each target sample.

4) Update the $\boldsymbol{\mu}_{c}^{t}$ according to the pseudo label of the target sample.

5) For $\left(k=0 ; k \leq \frac{\max \left(N_{s}, N_{t}\right)}{\text { batchsize }} ; k=k+1\right)$ do

a) Randomly sample a batch of labeled source domain instances and a batch of unlabeled target domain instances. Compute the classification loss and clustering loss using Equation 1 and 7.

b) For JCDFA-C, randomly augment the sampled source and target dataset, and store its affiliated label or pseudo label for the augmented sample.

c) Calculate the adaptation loss using the Equation 10 for JCDFA-C, or compute the the adaptation loss using the Equation 19 for JCDFA-M.

d) Back-propagate via the entire learning loss defined by the Equation 11 for JCDFA-C or the Equation 20 for JCDFA-M.

e) Update the network parameters $\boldsymbol{\theta}$ and $\phi$, and the clustering center $\boldsymbol{\mu}_{c}^{t}$.

End

End while

To adapt the discriminative features across domains, we further decrease the discrepancy of the class conditional distributions via an extended supervised contrastive learning and an extended MMD distance, respectively. Accordingly, the JCDFA-C and JCDFA-M are learned in detail.

1) JCDFA-C: The adaptation of the discriminative features step will explicitly minimize the distances of conditional distributions across domains and facilitate the adaptation of category-discriminative knowledge via the extended supervised contrastive learning. We expect the normalized embedding belonging to the same class to be close together, while those with different labels will be further away from each other.

Firstly, we extend the Supervised Contrastive learning (SupCon) loss [35] into the semi-supervised contrastive learning 
loss for UDA, which is defined as follows:

$$
\begin{aligned}
\mathcal{L}_{i}^{\text {intra }} & =\frac{-1}{2\left(N_{s}^{\tilde{y}_{i}}+N_{t}^{\tilde{y}_{i}}\right)-1} \cdot \frac{\tau_{0}}{\tau_{1}} \sum_{j=1}^{2\left(N_{s}+N_{t}\right)} \mathbf{1}_{i \neq j} \cdot \mathbf{1}_{\tilde{y}_{i}=\tilde{y}_{j}} \\
& \cdot \log \frac{\exp \left(\boldsymbol{v}_{i} \cdot \boldsymbol{v}_{i}\right)}{\sum_{k=1}^{2\left(N_{s}+N_{t}\right)} \mathbf{1}_{i \neq k} \cdot \exp \left(\boldsymbol{v}_{i} \cdot v_{k}\right)},
\end{aligned}
$$

where we conduct the cross-domain adaptation using the mixed dataset of two domains, so we get rid of the superscripts that specify the source domain and the target domain for simplicity. For example, we use $\tilde{y}_{i}$ to denote the true label of the source sample or the pseudo label of the target sample defined in Section III-B. Similarly, $\boldsymbol{v}_{i}=h\left(\boldsymbol{x}_{i} ; \boldsymbol{\theta}, \boldsymbol{\phi}\right)$ denotes the embedding of the source or target sample in the classifier output space. $N_{s}^{\tilde{y}_{i}}$ and $N_{t}^{\tilde{y}_{i}}$ are the total number of source and target images that have the same label $\tilde{y}_{i}$, respectively. $\tau_{0}$ and $\tau_{1}$ are applied to adjust the loss function, and set to 0.01 and 0.07 in all experiments, respectively. $\mathbf{1}_{i \neq j}$ and $\mathbf{1}_{\tilde{y}_{i}=\tilde{y}_{j}}$ are defined as follows: $\mathbf{1}_{i \neq j}=\left\{\begin{array}{lc}1 & \text { if } i \neq j \\ 0 & \text { otherwise }\end{array}\right.$, and $\mathbf{1}_{\tilde{y}_{i}=\tilde{y}_{j}}=$ $\left\{\begin{array}{ll}1 & \text { if } \tilde{y}_{i}=\tilde{y}_{j} \\ 0 & \text { otherwise }\end{array}\right.$. Equation 8 defines the discriminative feature alignment via cross-domain intra-class distances. In order to enhance the alignment of class-level distinguishing features, we further modify the proposed semi-supervised contrastive loss and add a new loss term, which ensures the maximum distance inter-class by pushing away from different classes of samples. The new added semi-supervised contrasive loss term via defining the inter-class distance can be written as follows:

$$
\begin{aligned}
& \mathcal{L}_{i}^{\text {inter }}=\frac{-1}{2\left(\breve{N}_{s}^{\tilde{y_{i}}}+\breve{N}_{t}^{\tilde{y}_{i}}\right)} \cdot \frac{\tau_{0}}{\tau_{1}} \sum_{j=1}^{2\left(N_{s}+N_{t}\right)} \mathbf{1}_{i \neq j} \cdot \mathbf{1}_{\tilde{y}_{i} \neq \tilde{y}_{j}} \\
& \cdot \log \frac{\exp \left(\boldsymbol{v}_{i} \cdot \boldsymbol{v}_{i}\right)}{\sum_{k=1}^{2\left(N_{s}+N_{t}\right)} \mathbf{1}_{i \neq k} \cdot \exp \left(\boldsymbol{v}_{i} \cdot v_{k}\right)},
\end{aligned}
$$

where $\breve{N}_{s}^{\tilde{y}_{i}}$ and $\breve{N}_{t}^{\tilde{y}_{i}}$ are the total number of source and target images whose labels are not $\tilde{y}_{i}$, respectively. $\mathbf{1}_{\tilde{y}_{i} \neq \tilde{y}_{j}}$ is defined as follows: $\mathbf{1}_{\tilde{y}_{i} \neq \tilde{y}_{j}}=\left\{\begin{array}{ll}1 & \text { if } \tilde{y}_{i} \neq \tilde{y}_{j} \\ 0 & \text { otherwise }\end{array}\right.$. We combine the Equation 8 and 9, the adaptation loss function of classlevel discriminative feature can be defined as follows:

$$
\mathcal{L}_{\text {ada }}(\boldsymbol{\theta}, \boldsymbol{\phi})=\sum_{i=1}^{2\left(N_{s}+N_{t}\right)}\left(\mathcal{L}_{i}^{\text {intra }}-\lambda_{0} \mathcal{L}_{i}^{\text {inter }}\right)
$$

where $\lambda_{0}$ is applied to balance the two loss terms. To be specific, we use twice the sum of the source domain and target domain samples, i.e., $2\left(N_{s}+N_{t}\right)$, because we make a random augmentation for each sample, which is the necessary step of the contrastive learning. In a whole, the first term of Equation 10 is designed to make the feature representations of samples more compact when the data pairs belong to the same class, while the second term pushes the feature representations of each other further away from the decision boundary when their labels are nonmatching.

By combining Equations 1, 7 and 10, the entire loss can thus be obtained:

$$
\mathcal{L}(\boldsymbol{\theta}, \boldsymbol{\phi})=\mathcal{L}_{c l s}(\boldsymbol{\theta}, \boldsymbol{\phi})+\lambda_{1} \mathcal{L}_{c l u}(\boldsymbol{\theta}, \boldsymbol{\phi})+\lambda_{2} \mathcal{L}_{a d a}(\boldsymbol{\theta}, \boldsymbol{\phi}),
$$

where $\lambda_{1}$ and $\lambda_{2}$ are applied to balance the loss function.

2) JCDFA-M: Similar to [61], [20], [62], we extend the Maximum Mean Discrepancy (MMD) [36] to class-level distribution discrepancy. We minimize the MMD distance of the intra-class sample pairs while maximizing the distance of the inter-class sample pairs in the probability output space. In more detail, the distance loss between two class conditional distributions with their mean embeddings in the reproducing kernel Hilbert space (RKHS) can be written as follows:

$$
\begin{aligned}
\mathcal{L}_{\text {ada }}^{(1)}(\boldsymbol{\theta}, \boldsymbol{\phi}) & =\frac{1}{C} \sum_{c=1}^{C}\left(\sum_{\boldsymbol{x}_{i} \in X_{(c)}} \sum_{\boldsymbol{x}_{j} \in X_{(c)}}\left\|\psi\left(\boldsymbol{v}_{i}\right)-\psi\left(\boldsymbol{v}_{j}\right)\right\|_{\mathcal{H}}\right. \\
& \left.-\sum_{\boldsymbol{x}_{i} \in X_{(c)}} \sum_{\boldsymbol{x}_{u} \notin X_{(c)}}\left\|\psi\left(\boldsymbol{v}_{i}\right)-\psi\left(\boldsymbol{v}_{u}\right)\right\|_{\mathcal{H}}\right),
\end{aligned}
$$

where $X_{(c)}=X_{(c)}^{s} \cup \hat{X}_{(c)}^{t}$, while $X_{(c)}^{s}$ denotes source samples in class $c . \hat{X}_{(c)}^{t}$ denotes target samples in pseudo-class $c . \psi(\cdot)$ is the kernel feature map of RKHS. As computing the Equation 12 directly is intractable, we use the kernel trick to rewrite the first term as follows:

$$
\begin{aligned}
d_{\text {intra }}^{(1)} & =\frac{1}{C} \sum_{c=1}^{C}\left(o_{1}+o_{2}-2 o_{3}\right), \\
o_{1} & =\sum_{i=1}^{N_{s}} \sum_{j=1}^{N_{s}} \frac{\mathbf{1}_{c}\left(y_{i}^{s}, y_{j}^{s}\right) k\left(\boldsymbol{v}_{i}^{s}, \boldsymbol{v}_{j}^{s}\right)}{\sum_{i=1}^{N_{s}} \sum_{j=1}^{N_{s}} \mathbf{1}_{c}\left(y_{i}^{s}, y_{j}^{s}\right)}, \\
o_{2} & =\sum_{i=1}^{N_{t}} \sum_{j=1}^{N_{t}} \frac{\mathbf{1}_{c}\left(\hat{y}_{i}^{t}, \hat{y}_{j}^{t}\right) k\left(\boldsymbol{v}_{i}^{t}, \boldsymbol{v}_{j}^{t}\right)}{\sum_{i=1}^{N_{t}} \sum_{j=1}^{N_{t}} \mathbf{1}_{c}\left(\hat{y}_{i}^{t}, \hat{y}_{j}^{t}\right)}, \\
o_{3}= & \sum_{i=1}^{N_{s}} \sum_{j=1}^{N_{t}} \frac{\mathbf{1}_{c}\left(y_{i}^{s}, \hat{y}_{j}^{t}\right) k\left(\boldsymbol{v}_{i}^{s}, \boldsymbol{v}_{j}^{t}\right)}{\sum_{i=1}^{N_{s}} \sum_{j=1}^{N_{t}} \mathbf{1}_{c}\left(y_{i}^{s}, \hat{y}_{j}^{t}\right)},
\end{aligned}
$$

where $\hat{y}_{i}^{t}$ and $\hat{y}_{j}^{t}$ denote the pseudo-labels of the target domain predicted defined in Section III-B, while $k$ represents the kernel function [18]. Each element of $\mathbf{1}_{c}\left(y, y^{\prime}\right)$ is defined as: $\mathbf{1}_{c}\left(y, y^{\prime}\right)=1$ if $y=y^{\prime}=c ; \mathbf{1}_{c}\left(y, y^{\prime}\right)=0$, otherwise. In the same way, the second term of Equation 12 can be written as follows:

$$
\begin{aligned}
d_{\text {inter }}^{(1)} & =\frac{1}{C(C-1)} \sum_{c=1}^{C} \sum_{c^{\prime}=1, c^{\prime} \neq c}^{C}\left(o_{1}^{\prime}+o_{2}^{\prime}-2 o_{3}^{\prime}\right), \\
o_{1}^{\prime} & =\sum_{i=1}^{N_{s}} \sum_{j=1}^{N_{s}} \frac{\mathbf{1}_{c c}\left(y_{i}^{s}, y_{j}^{s}\right) k\left(\boldsymbol{v}_{i}^{s}, \boldsymbol{v}_{j}^{s}\right)}{\sum_{i=1}^{N_{s}} \sum_{j=1}^{N_{s}} \mathbf{1}_{c c}\left(y_{i}^{s}, y_{j}^{s}\right)}, \\
o_{2}^{\prime} & =\sum_{i=1}^{N_{t}} \sum_{j=1}^{N_{t}} \frac{\mathbf{1}_{c^{\prime} c^{\prime}}\left(\hat{y}_{i}^{t}, \hat{y}_{j}^{t}\right) k\left(\boldsymbol{v}_{i}^{t}, \boldsymbol{v}_{j}^{t}\right)}{\sum_{i=1}^{N_{t}} \sum_{j=1}^{N_{t}} \mathbf{1}_{c^{\prime} c^{\prime}}\left(\hat{y}_{i}^{t}, \hat{y}_{j}^{t}\right)}, \\
o_{3}^{\prime} & =\sum_{i=1}^{N_{s}} \sum_{j=1}^{N_{t}} \frac{\mathbf{1}_{c c^{\prime}}\left(y_{i}^{s}, \hat{y}_{j}^{t}\right) k\left(\boldsymbol{v}_{i}^{s}, \boldsymbol{v}_{j}^{t}\right)}{\sum_{i=1}^{N_{s}} \sum_{j=1}^{N_{t}} \mathbf{1}_{c c^{\prime}}\left(y_{i}^{s}, \hat{y}_{j}^{t}\right)},
\end{aligned}
$$

where the element of $\mathbf{1}_{c c^{\prime}}\left(y, y^{\prime}\right)$ is defined as: $\mathbf{1}_{c c^{\prime}}\left(y, y^{\prime}\right)=1$, if $y=c, y^{\prime}=c^{\prime} ; \mathbf{1}_{c c^{\prime}}\left(y, y^{\prime}\right)=0$, otherwise. By Combining Equations 13 and 14, Equation 12 can be rewritten as follows:

$$
\mathcal{L}_{\text {ada }}^{(1)}(\boldsymbol{\theta}, \boldsymbol{\phi})=d_{\text {intra }}^{(1)}-d_{\text {inter }}^{(1)},
$$

To strengthen the transferability of the class discriminative features, we further introduce the adaptation of class-level 
distribution in the last layer output of the feature extractor. We conduct the adaptation learning of the latent feature $z$ :

$$
\mathcal{L}_{\text {ada }}^{(2)}(\boldsymbol{\theta})=d_{\text {intra }}^{(2)}-d_{\text {inter }}^{(2)}
$$

where $d_{\text {intra }}^{(2)}$ is defined as follows:

$$
d_{\text {intra }}^{(2)}=\frac{1}{C} \sum_{c=1}^{C}\left(\hat{o}_{1}+\hat{o}_{2}-2 \hat{o}_{3}\right)
$$

where the definition $\hat{o}$. is similar to Equation 13, e.g., $\hat{o}_{1}=$ $\sum_{i=1}^{N_{s}} \sum_{j=1}^{N_{s}} \frac{\left.\mathbf{1}_{c}\left(y_{i}^{s}, y_{j}^{s}\right) k\left(z_{i}^{s}, z_{j}^{s}\right)\right)}{\sum_{i=1}^{N_{s}} \sum_{j=1}^{N_{s}} \mathbf{1}_{c}\left(y_{i}^{s}, y_{j}^{s}\right)}$.

Moreover the $d_{\text {inter }}^{(2)}$ is defined as follows:

$$
d_{\text {inter }}^{(2)}=\frac{1}{C(C-1)} \sum_{c=1}^{C} \sum_{c^{\prime}=1, c^{\prime} \neq c}^{C}\left(\hat{o}_{1}^{\prime}+\hat{o}_{2}^{\prime}-2 \hat{o}_{3}^{\prime}\right),
$$

where the definition $\hat{o}^{\prime}$ is similar to Equation 14, e.g., $\hat{o}_{1}^{\prime}=$ $\sum_{i=1}^{N_{s}} \sum_{j=1}^{N_{s}} \frac{\mathbf{1}_{c c^{\prime}}\left(y_{i}^{s}, \dot{y}_{j}^{s}\right) k\left(z_{i}^{s}, z_{j}^{s}\right)}{\sum_{i=1}^{N_{s}} \sum_{j=1}^{N_{s}} \mathbf{1}_{c c^{\prime}}\left(y_{i}^{s}, y_{j}^{s}\right)}$.

By combining Equations 15 and 16, we can obtain the overall loss of the conditional distributions:

$$
\mathcal{L}_{\text {ada }}^{\prime}(\boldsymbol{\theta}, \boldsymbol{\phi})=\mathcal{L}_{\text {ada }}^{(1)}(\boldsymbol{\theta}, \boldsymbol{\phi})+\mathcal{L}_{\text {ada }}^{(2)}(\boldsymbol{\theta})
$$

The entire loss can thus be obtained via the equation:

$$
\mathcal{L}(\boldsymbol{\theta}, \boldsymbol{\phi})=\mathcal{L}_{\text {cls }}(\boldsymbol{\theta}, \boldsymbol{\phi})+\lambda_{1}^{\prime} \mathcal{L}_{c l u}(\boldsymbol{\theta}, \boldsymbol{\phi})+\lambda_{2}^{\prime} \mathcal{L}_{\text {ada }}^{\prime}(\boldsymbol{\theta}, \boldsymbol{\phi}),
$$

where $\lambda_{1}^{\prime}$ and $\lambda_{2}^{\prime}$ are applied to balance the loss function.

For clarity, we sketch the main steps of our proposed JCDFA in Algorithm 1.

\section{EXPERIMENTS}

\section{A. Datasets}

We evaluate our proposed JCDFA on four UDA datasets: Office-31 [64], ImageCLEF-DA ${ }^{2}$, Office-Home [65] and VisDA-C [66]. Figure 4 shows examples of these four datasets. For each dataset, each row represents one domain.

Office-31 which is a standard dataset used to evaluate different DA methods for object recognition, consists of three different domains: Amazon (A), Dslr (D), and Webcam (W), and includes 4,652 images in 31 classes. Amazon images are collected from amazon.com, while Webcam and Dslr images are taken using a webcam and a high-quality camera, respectively.

ImageCLEF-DA is a benchmark dataset for ImageCLEF 2014 domain adaptation challenge, which is organized by selecting the 12 common classes shared by three public datasets (domains): Caltech-256 (C), ImageNet ILSVRC 2012 (I), and Pascal VOC 2012 (P). For each domain, there are 50 images in each category. Each dataset contains 6 domain adaptation tasks.

Office-Home is a large benchmark dataset containing around 15,500 images divided into 65 classes. The dataset comprises four domains: Artistic (Ar), Clip Art (Cl), Product (Pr) and Real-World (Rw).

VisDA-C is a highly challenging dataset with the domain shift from synthetic data to real imagery. It has two domains

\footnotetext{
${ }^{2}$ http://imageclef.org/2014/adaptation
}

where the Synthetic one, consisting of 152,397 synthetic 2D renderings of $3 \mathrm{D}$ objects, and the Real one, consisting of 55,388 real images cropped from the MS-COCO [67] dataset. These two domains have 12 classes in common.

\section{B. Implementation Details}

We applied ResNet-50 [63], pretrained on ImageNet [68] as the feature extractor branch, and replaced the last FC layer with the task-specific FC layer.

We implemented all experiments using Pytorch [69]. The network was trained using the mini-batch stochastic gradient descent (SGD) optimizer with momentum of 0.9. Following CAN [20], we use class-aware sampling strategy to train the model. In detail, we randomly select a subset of classes from all categories, and then sample source data and target data for each class. The sampled source data and target data consist of each batch of data, respectively. Thus, using each batch of training data, we are able to compute the intraclass and inter-class discrepancy for each selected class. The learning rate annealing strategy was adopted as [29], [20]: $\eta_{p}=\eta_{0}(1+\alpha p)^{-\beta}$, where $p$ denotes the training progress changing from 0 to the maximum number of iterations. For Office-31, ImageCLEF-DA and Office-Home, $\alpha=0.001$, $\beta=0.75$, while for VisDA-C, $\alpha=0.001$ and $\beta=2.25$. $\eta_{0}$ indicates the initial learning rate, i.e. $1 \mathrm{e}-3$ for the convolutional layers and 1e-2 for the task-specific FC layer.

For JCDFA-C, the tradeoff parameter $\lambda_{0}$ is set to 0.01 and 0.1 for $\mathrm{A} \rightarrow \mathrm{W}$ and $\mathrm{A} \rightarrow \mathrm{D}$ tasks, respectively, and set to 1 for other tasks. We increase $\lambda_{1}$ from 0 to 1 via $\lambda_{1}=2(1+$ $\exp (-\gamma \tilde{p}))^{-1}-1$ following the [27], [49], where $\gamma$ is set to 10 and $\tilde{p}$ denotes the training process normalized to be in $[0,1]$. The $\lambda_{2}$ is set to 0.3 based on the accurate model selection [70].

For JCDFA-M, the tradeoff parameter $\lambda_{1}^{\prime}$ annealing strategy is $\lambda_{1}^{\prime}={\hat{\lambda^{\prime}}}_{1}(1+0.001 * p)^{-0.75}$, where $\hat{\lambda}^{\prime}{ }_{1}$ is set to 0.1 . The $\lambda_{2}^{\prime}$ is set to 0.3 following the hyperparameter selection strategy [70]. We used a linear combination of 5 Gaussian kernels for MMD.

In particular, for the target domain, we concentrated only on the data with high reliability; i.e., we filtered out ambiguous data points that are located far from the corresponding cluster centroid. Similar to CAN [20], the point-to-centroid threshold is defined as 0.05 for Office-31 tasks $\mathrm{A} \rightarrow \mathrm{W}, \mathrm{D} \rightarrow \mathrm{W}, \mathrm{W} \rightarrow$ $\mathrm{D}$ and $\mathrm{A} \rightarrow \mathrm{D}$. For other tasks, this threshold is defined as 1 .

\section{Results}

Results on Office-31 We first carried out experiments on the Office-31 dataset following the fully transductive evaluation protocol [26]. To facilitate fair comparison, the results of other comparison methods are directly quoted from their original papers. Experimental results are presented in Table I. The first row lists results without any adaptation, which acts as the lower bound. From results comparison, we can observe that our proposed method JCDFA-M outperforms state-ofthe-art methods on all transfer tasks, which strongly confirms the effectiveness of our proposed JCDFA-M in mining the transferable discriminative features. It is noteworthy that 

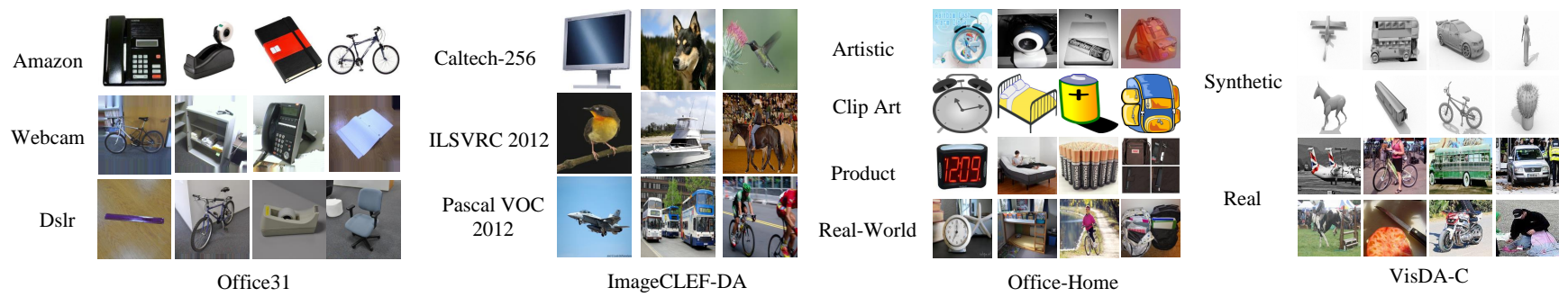

Fig. 4. Examples from the Office-31, ImageCLEF-DA, Office-Home and VisDA-C datasets used in our experiments. For each dataset, each row denotes a domain.

TABLE I

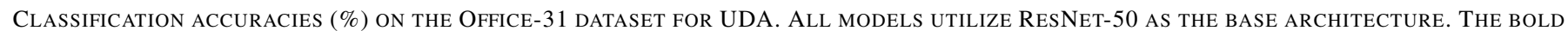
NUMBERS DENOTE THE BEST RESULTS FOR EACH COLUMN.

\begin{tabular}{llllllll}
\hline & $\mathrm{A} \rightarrow \mathrm{W}$ & $\mathrm{D} \rightarrow \mathrm{W}$ & $\mathrm{W} \rightarrow \mathrm{D}$ & $\mathrm{A} \rightarrow \mathrm{D}$ & $\mathrm{D} \rightarrow \mathrm{A}$ & $\mathrm{W} \rightarrow \mathrm{A}$ & Average \\
\hline ResNet-50 [63] & $77.8 \pm 0.2$ & $96.9 \pm 0.1$ & $99.3 \pm 0.1$ & $82.1 \pm 0.2$ & $64.5 \pm 0.2$ & $66.1 \pm 0.2$ & 81.1 \\
DAN [18] & $81.3 \pm 0.3$ & $97.2 \pm 0.0$ & $99.8 \pm 0.0$ & $83.1 \pm 0.2$ & $66.3 \pm 0.0$ & $66.3 \pm 0.1$ & 82.3 \\
DANN [27] & $81.7 \pm 0.2$ & $98.0 \pm 0.2$ & $99.8 \pm 0.0$ & $83.9 \pm 0.7$ & $66.4 \pm 0.2$ & $66.0 \pm 0.3$ & 82.6 \\
JAN [21] & $85.4 \pm 0.3$ & $97.4 \pm 0.2$ & $99.8 \pm 0.2$ & $84.7 \pm 0.3$ & $68.6 \pm 0.3$ & $70.0 \pm 0.4$ & 84.3 \\
SimNet [52] & $88.6 \pm 0.5$ & $98.2 \pm 0.2$ & $99.7 \pm 0.2$ & $85.3 \pm 0.3$ & $73.4 \pm 0.8$ & $71.8 \pm 0.6$ & 86.2 \\
CAT [46] & $91.1 \pm 0.2$ & $98.6 \pm 0.6$ & $99.6 \pm 0.1$ & $90.6 \pm 1.0$ & $70.4 \pm 0.7$ & $66.5 \pm 0.4$ & 86.1 \\
MCD [30] & $88.6 \pm 0.2$ & $98.5 \pm 0.1$ & $\mathbf{1 0 0 . 0} \pm 0.0$ & $92.2 \pm 0.2$ & $69.5 \pm 0.1$ & $69.7 \pm 0.3$ & 86.5 \\
MSTN [51] & 91.3 & 98.9 & $\mathbf{1 0 0 . 0}$ & 90.4 & 72.7 & 65.6 & 86.5 \\
DWL [44] & 89.2 & 99.2 & 100.0 & 91.2 & 73.1 & 69.8 & 87.1 \\
CDAN+E [29] & $94.1 \pm 0.1$ & $98.6 \pm 0.1$ & $\mathbf{1 0 0 . 0} \pm 0.0$ & $92.9 \pm 0.2$ & $71.0 \pm 0.3$ & $69.3 \pm 0.3$ & 87.7 \\
TAT [42] & $92.5 \pm 0.3$ & $99.3 \pm 0.1$ & $\mathbf{1 0 0 . 0} \pm 0.0$ & $93.2 \pm 0.2$ & $73.1 \pm 0.3$ & $72.1 \pm 0.3$ & 88.4 \\
SymNets [41] & $90.8 \pm 0.1$ & $98.8 \pm 0.3$ & $\mathbf{1 0 0 . 0} \pm 0.0$ & $93.9 \pm 0.5$ & $74.6 \pm 0.6$ & $72.5 \pm 0.5$ & 88.4 \\
GSDA [50] & 95.7 & 99.1 & $\mathbf{1 0 0 . 0}$ & 94.8 & 73.5 & 74.9 & 89.7 \\
RSDA-DANN [43] & $95.3 \pm 0.3$ & $\mathbf{9 9 . 3} \pm 0.2$ & $\mathbf{1 0 0 . 0} \pm 0.0$ & $95.2 \pm 0.2$ & $75.5 \pm 0.6$ & $76.0 \pm 0.6$ & 90.2 \\
CAN [20] & $94.5 \pm 0.3$ & $99.1 \pm 0.2$ & $99.8 \pm 0.2$ & $95.0 \pm 0.3$ & $78.0 \pm 0.3$ & $77.0 \pm 0.3$ & 90.6 \\
SRDC [49] & $95.7 \pm 0.2$ & $99.2 \pm 0.1$ & $\mathbf{1 0 0 . 0} \pm 0.0$ & $\mathbf{9 5 . 8} \pm 0.2$ & $76.7 \pm 0.3$ & $77.1 \pm 0.1$ & 90.8 \\
\hline JCDFA-C & $94.2 \pm 0.2$ & $99.1 \pm 0.1$ & $\mathbf{1 0 0 . 0} \pm 0.0$ & $95.4 \pm 0.2$ & $77.1 \pm 0.3$ & $76.5 \pm 0.2$ & 90.4 \\
JCDFA-M & $\mathbf{9 6 . 1} \pm 0.3$ & $99.2 \pm 0.0$ & $\mathbf{1 0 0 . 0} \pm 0.0$ & $95.5 \pm 0.2$ & $\mathbf{7 9 . 2} \pm 0.3$ & $\mathbf{7 8 . 1} \pm 0.2$ & $\mathbf{9 1 . 4}$ \\
\hline
\end{tabular}

TABLE II

Classification aCcuracies (\%) on the ImageCLEF-DA dataset For UDA. All Models utilize ResNet-50 as the Base arChitecture. THE BOLD NUMBERS DENOTE THE BEST RESULTS FOR EACH COLUMN.

\begin{tabular}{llllllll}
\hline & $\mathrm{I} \rightarrow \mathrm{P}$ & $\mathrm{P} \rightarrow \mathrm{I}$ & $\mathrm{I} \rightarrow \mathrm{C}$ & $\mathrm{C} \rightarrow \mathrm{I}$ & $\mathrm{C} \rightarrow \mathrm{P}$ & $\mathrm{P} \rightarrow \mathrm{C}$ & Average \\
\hline ResNet-50 [63] & $74.8 \pm 0.3$ & $83.9 \pm 0.1$ & $91.5 \pm 0.3$ & $78.0 \pm 0.2$ & $65.5 \pm 0.3$ & $91.2 \pm 0.3$ & 80.7 \\
DAN [18] & $74.5 \pm 0.4$ & $82.2 \pm 0.2$ & $92.8 \pm 0.2$ & $86.3 \pm 0.4$ & $69.2 \pm 0.4$ & $89.8 \pm 0.4$ & 82.5 \\
DANN [27] & $75.0 \pm 0.6$ & $86.0 \pm 0.3$ & $96.2 \pm 0.4$ & $87.0 \pm 0.5$ & $74.3 \pm 0.5$ & $91.5 \pm 0.6$ & 85.0 \\
JAN [21] & $76.8 \pm 0.4$ & $88.0 \pm 0.2$ & $94.7 \pm 0.2$ & $89.5 \pm 0.3$ & $74.2 \pm 0.3$ & $91.7 \pm 0.3$ & 85.8 \\
CAT [46] & $76.7 \pm 0.2$ & $89.0 \pm 0.7$ & $94.5 \pm 0.4$ & $89.8 \pm 0.3$ & $74.0 \pm 0.2$ & $93.7 \pm 1.0$ & 86.3 \\
CDAN+E [29] & $77.7 \pm 0.3$ & $90.7 \pm 0.2$ & $97.7 \pm 0.3$ & $91.3 \pm 0.3$ & $74.2 \pm 0.2$ & $94.3 \pm 0.3$ & 87.7 \\
TAT [42] & $78.8 \pm 0.2$ & $92.0 \pm 0.2$ & $97.5 \pm 0.3$ & $92.0 \pm 0.3$ & $78.2 \pm 0.4$ & $94.7 \pm 0.4$ & 88.9 \\
SymNets [41] & $\mathbf{8 0 . 2} \pm 0.3$ & $93.6 \pm 0.2$ & $97.0 \pm 0.3$ & $93.4 \pm 0.3$ & $\mathbf{7 8 . 7} \pm 0.3$ & $96.4 \pm 0.1$ & 89.9 \\
RSDA-DANN [43] & $79.2 \pm 0.4$ & $93.0 \pm 0.2$ & $\mathbf{9 8 . 3} \pm 0.4$ & $93.6 \pm 0.4$ & $78.5 \pm 0.3$ & $\mathbf{9 8 . 2} \pm 0.2$ & 90.1 \\
DWL [44] & 82.3 & $\mathbf{9 4 . 8}$ & 98.1 & 92.8 & 77.9 & 97.2 & $\mathbf{9 0 . 5}$ \\
\hline JCDFA-C & $79.4 \pm 0.3$ & $94.5 \pm 0.2$ & $98.0 \pm 0.3$ & $\mathbf{9 3 . 7} \pm 0.3$ & $78.5 \pm 0.2$ & $97.3 \pm 0.2$ & 90.2 \\
JCDFA-M & $78.9 \pm 0.3$ & $94.1 \pm 0.2$ & $97.9 \pm 0.1$ & $93.5 \pm 0.3$ & $78.3 \pm 0.1$ & $97.3 \pm 0.2$ & 90.0 \\
\hline
\end{tabular}

JCDFA-M substantially promotes the classification accuracy on the hard transfer task $\mathrm{D} \rightarrow \mathrm{A}$. Although the proposed JCDFA-C can not achieve the state-of-the-art performance, the JCDFA-C does not introduce kernel computing, which reduces the computational complexity. Notably, JCDFA-M exceeds the excellent results obtained by CAN [20] in terms of its ability demonstrating that clustering learning could promote the following of following class-level feature adaptation.
Results on ImageCLEF-DA Results on ImageCLEF-DA are reported in Table II. JCDFA-C and JCDFA-M achieve better performance compared to most comparison methods expect RSDA-DANN [43] and DWL [44]. RSDA-DANN can obtain better performance due to ensembling RSDA and DANN. The best performance is achieved by DWL owe to weighting the samples according to the imbalance degree of the sample number of the two domains. JCDFA-C performs 
TABLE III

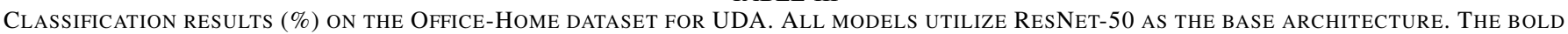
NUMBERS DENOTE THE BEST RESULTS FOR EACH COLUMN.

\begin{tabular}{|c|c|c|c|c|c|c|c|c|c|c|c|c|c|}
\hline & $\mathrm{Ar} \rightarrow \mathrm{Cl}$ & $\mathrm{Ar} \rightarrow \mathrm{Pr}$ & $\mathrm{Ar} \rightarrow \mathrm{Rw}$ & $\mathrm{Cl} \rightarrow \mathrm{Ar}$ & $\mathrm{Cl} \rightarrow \mathrm{Pr}$ & $\mathrm{Cl} \rightarrow \mathrm{Rw}$ & $\mathrm{Pr} \rightarrow \mathrm{Ar}$ & $\mathrm{Pr} \rightarrow \mathrm{Cl}$ & $\mathrm{Pr} \rightarrow \mathrm{RW}$ & $\mathrm{Rw} \rightarrow \mathrm{Ar}$ & $\mathrm{Rw} \rightarrow \mathrm{Cl}$ & $\mathrm{Rw} \rightarrow \mathrm{Pr}$ & Ave. \\
\hline ResNet-50 [63] & 34.9 & 50.0 & 58.0 & 37.4 & 41.9 & 46.2 & 38.5 & 31.2 & 60.4 & 53.9 & 41.2 & 59.9 & 46.1 \\
\hline DAN [18] & 43.6 & 57.0 & 67.9 & 45.8 & 56.5 & 60.4 & 44.0 & 43.6 & 67.7 & 63.1 & 51.5 & 74.3 & 56.3 \\
\hline DANN [27] & 45.6 & 59.3 & 70.1 & 47.0 & 58.5 & 60.9 & 46.1 & 43.7 & 68.5 & 63.2 & 51.8 & 76.8 & 57.6 \\
\hline JAN [21] & 45.9 & 61.2 & 68.9 & 50.4 & 59.7 & 61.0 & 45.8 & 43.4 & 70.3 & 63.9 & 52.4 & 76.8 & 58.3 \\
\hline DWT-MEC [50] & 50.3 & 72.1 & 77.0 & 59.6 & 69.3 & 70.2 & 58.3 & 48.1 & 77.3 & 69.3 & 53.6 & 82.0 & 65.6 \\
\hline $\mathrm{CDAN}+\mathrm{E}$ [29] & 50.7 & 70.6 & 76.0 & 57.6 & 70.0 & 70.0 & 57.4 & 50.9 & 77.3 & 69.3 & 53.6 & 82.0 & 65.8 \\
\hline TAT [42] & 51.6 & 69.5 & 75.4 & 59.4 & 69.5 & 68.6 & 59.5 & 50.5 & 76.8 & 70.9 & 56.6 & 81.6 & 65.8 \\
\hline SymNets [41] & 47.7 & 72.9 & 78.5 & 64.2 & 71.3 & 74.2 & 64.2 & 48.8 & 79.5 & 74.5 & 52.6 & 82.7 & 67.6 \\
\hline RSDA-DANN [43] & 51.5 & 76.8 & 81.1 & 67.1 & 72.1 & 77.0 & 64.2 & 51.1 & 81.8 & 74.9 & 55.9 & 84.5 & 69.8 \\
\hline GSDA [50] & 61.3 & 76.1 & 79.4 & 65.4 & 73.3 & 74.3 & 65.0 & 53.2 & 80.0 & 72.2 & 60.6 & 83.1 & 70.3 \\
\hline SRDC [49] & 52.3 & 76.3 & 81.0 & 69.5 & 76.2 & 78.0 & 68.7 & 53.8 & 81.7 & 76.3 & 57.1 & 85.0 & 71.3 \\
\hline JCDFA-C & 60.7 & 79.8 & 82.1 & 69.6 & 75.4 & 77.5 & 68.5 & 56.0 & 82.7 & 73.8 & 61.0 & 82.9 & 72.5 \\
\hline JCDFA-M & 61.7 & 78.1 & 83.2 & 70.1 & 77.9 & 78.6 & 69.8 & 59.7 & 83.4 & 75.9 & 63.4 & 85.9 & 74.0 \\
\hline
\end{tabular}

TABLE IV

Classification accuracies (\%) ON THE VisDA-C DATASET For UDA. All Models Utilize ResNet-50 as the Base ARChitecture. The Bold NUMBERS DENOTE THE BEST RESULT.

\begin{tabular}{|l|c|ccccccccc|}
\hline Methods & ResNet-50 [63] & DAN [18] & DANN [27] & JAN [21] & MCD [30] & SimNet [52] & CDAN+E [29] & TAT [42] & JCDFA-C & JCDFA-M \\
\hline Average & 60.0 & 63.1 & 63.7 & 64.8 & 69.2 & 69.6 & 70.0 & 71.9 & $\mathbf{7 8 . 9}$ & 75.4 \\
\hline
\end{tabular}
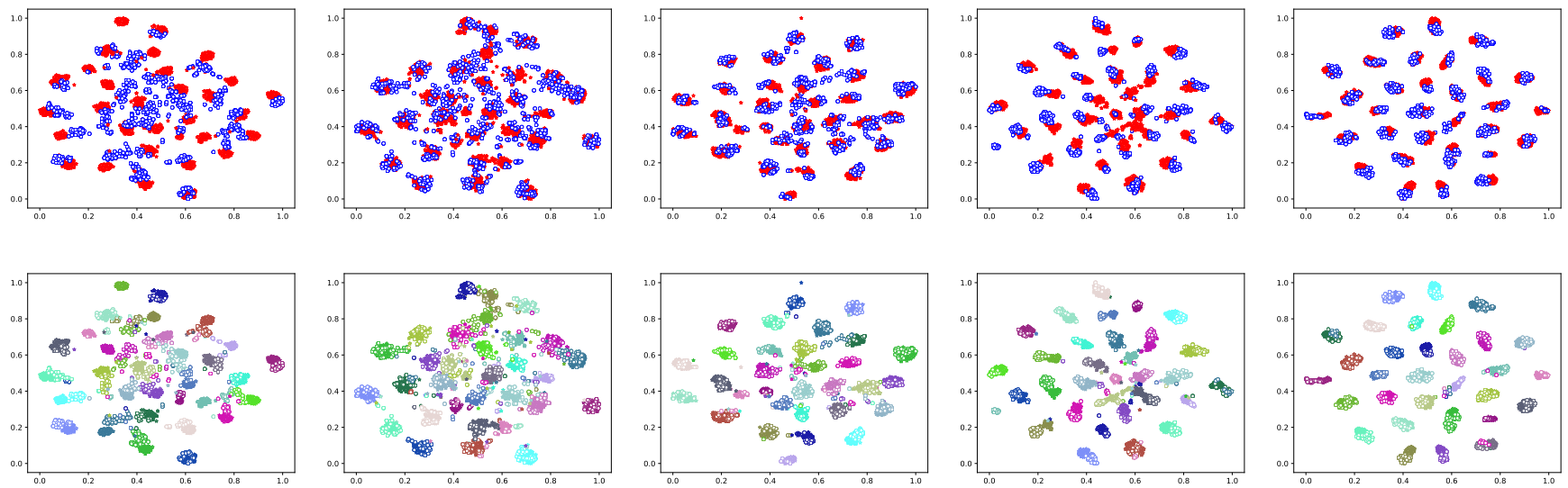

(a) ResNet-50

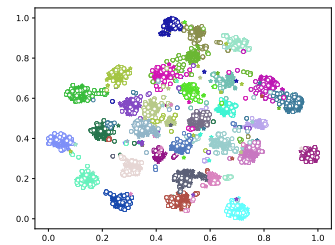

(b) DANN

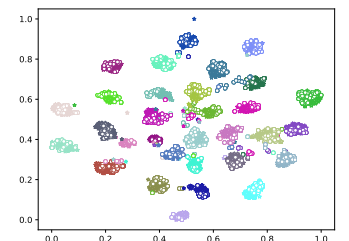

(c) CDAN

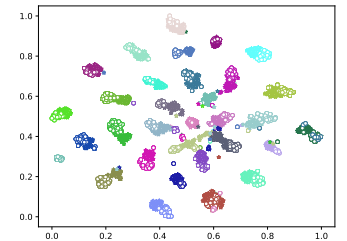

(d) JCDFA-C

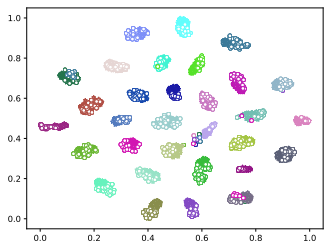

(e) JCDFA-M

Fig. 5. (a)-(e) The t-SNE visualization of embedded features generated by ResNet-50, DANN, CDAN, JCDFA-C and JCDFA-M on the task A $\rightarrow$ W, respectively. The "*" and "o" represent the source and target domain, respectively. In the first row, different colors represent different domains. In the second row, different colors represent different classes.

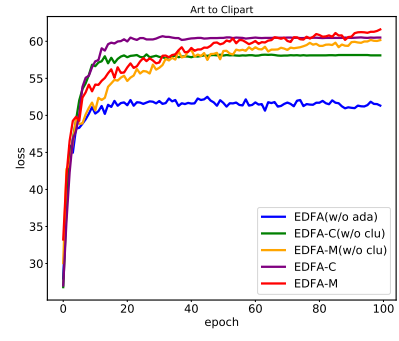

(a) $\mathrm{Ar} \rightarrow \mathrm{Cl}$

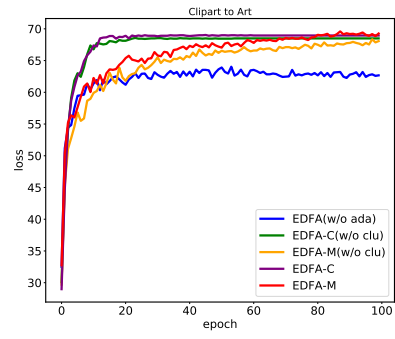

(b) $\mathrm{Cl} \rightarrow \mathrm{Ar}$

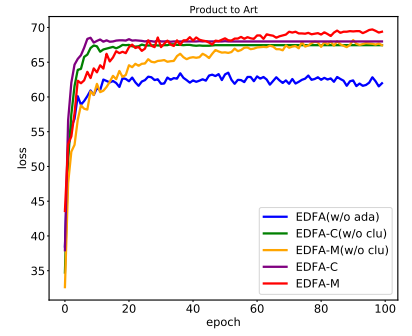

(c) $\mathrm{Pr} \rightarrow \mathrm{Ar}$

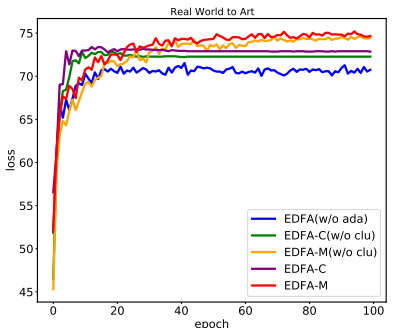

(d) $\mathrm{Rw} \rightarrow \mathrm{Ar}$

Fig. 6. (a)-(d) Accuracy curves of different methods on the task $\mathrm{Ar} \rightarrow \mathrm{Cl}, \mathrm{Cl} \rightarrow \mathrm{Ar}, \mathrm{Pr} \rightarrow \mathrm{Ar}$ and $\mathrm{Rw} \rightarrow \mathrm{Ar}$.

slightly better than JCDFA-M on all transfer tasks, verifying the efficacy of semi-supervised contrastive learning in the context of category balance.
Results on Office-Home Table III illustrates the classification accuracies of experimental methods on the challenging Office-Home dataset. This table reveals that, as desired, 
TABLE V

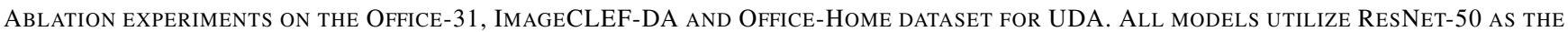
BASE ARCHITECTURE. THE BOLD NUMBERS DENOTE THE BEST RESULTS FOR EACH COLUMN IN EACH BOX.

\begin{tabular}{|c|c|c|c|c|c|c|c|c|c|c|c|c|c|}
\hline & \multicolumn{2}{|c|}{$\mathrm{A} \rightarrow \mathrm{W}$} & \multicolumn{2}{|c|}{$\mathrm{D} \rightarrow \mathrm{W}$} & \multicolumn{2}{|c|}{$\mathrm{W} \rightarrow \mathrm{D}$} & \multicolumn{2}{|c|}{$\mathrm{A} \rightarrow \mathrm{D}$} & \multicolumn{2}{|c|}{$\mathrm{D} \rightarrow \mathrm{A}$} & \multicolumn{2}{|c|}{$\mathrm{W} \rightarrow \mathrm{A}$} & Ave. \\
\hline JCDFA (w/o ada) & \multicolumn{2}{|c|}{$84.9 \pm 0.1$} & \multicolumn{2}{|c|}{$98.2 \pm 0.1$} & \multicolumn{2}{|c|}{$100.0 \pm 0.0$} & \multicolumn{2}{|c|}{$84.7 \pm 0.1$} & \multicolumn{2}{|c|}{$75.3 \pm 0.3$} & \multicolumn{2}{|c|}{$73.0 \pm 0.1$} & 86.0 \\
\hline JCDFA-C (w/o inter) & \multicolumn{2}{|c|}{$94.1 \pm 0.2$} & \multicolumn{2}{|c|}{$97.9 \pm 0.2$} & \multicolumn{2}{|c|}{$100.0 \pm 0.0$} & \multicolumn{2}{|c|}{$94.3 \pm 0.2$} & \multicolumn{2}{|c|}{$76.1 \pm 0.2$} & \multicolumn{2}{|c|}{$75.8 \pm 0.1$} & 89.7 \\
\hline JCDFA-C (w/o clu) & \multicolumn{2}{|c|}{$94.0 \pm 0.1$} & \multicolumn{2}{|c|}{$98.2 \pm 0.2$} & \multicolumn{2}{|c|}{$100.0 \pm 0.0$} & \multicolumn{2}{|c|}{$94.1 \pm 0.2$} & \multicolumn{2}{|c|}{$76.4 \pm 0.2$} & \multicolumn{2}{|c|}{$76.2 \pm 0.3$} & 89.8 \\
\hline JCDFA-C & \multicolumn{2}{|c|}{$94.2 \pm 0.2$} & \multicolumn{2}{|c|}{$99.1 \pm 0.1$} & \multicolumn{2}{|c|}{$100.0 \pm 0.0$} & \multicolumn{2}{|c|}{$95.4 \pm 0.2$} & \multicolumn{2}{|c|}{$77.1 \pm 0.3$} & \multicolumn{2}{|c|}{$76.5 \pm 0.2$} & 90.4 \\
\hline JCDFA-M (w/o clu) & \multicolumn{2}{|c|}{$94.5 \pm 0.3$} & \multicolumn{2}{|c|}{$99.1 \pm 0.2$} & & \pm 0.2 & 95.0 & 0.3 & 78. & $=0.3$ & 77. & 0.3 & 90.6 \\
\hline JCDFA-M & 96.1 & $=0.3$ & 99.2 & 0.0 & 100 & \pm 0.0 & 95.5 & 0.2 & 79.2 & \pm 0.3 & 78.1 & \pm 0.2 & 91.4 \\
\hline & & & & & & & & & & & & & Ave. \\
\hline JCDFA (w/o ada) & 78. & 0.2 & 92.7 & & & \pm 0.1 & 91.2 & 0.1 & 77. & 0.3 & 95. & 0.1 & 88.7 \\
\hline JCDFA-C (w/o inter) & 78.2 & 0.1 & 93.3 & & & \pm 0.1 & 93.1 & 0.2 & 77. & 0.1 & $97 .($ & 0.2 & 89.2 \\
\hline JCDFA-C (w/o clu) & 78. & 0.2 & 94.1 & & & \pm 0.3 & 92.7 & 0.2 & 76. & $=0.1$ & 97.3 & $=0.2$ & 89.4 \\
\hline JCDFA-C & 79.4 & $=0.3$ & 94.5 & 0.2 & & \pm 0.3 & 93.7 & 0.3 & 78.5 & \pm 0.2 & 97.3 & \pm 0.2 & 90.2 \\
\hline JCDFA-M (w/o clu) & 78.2 & $=0.3$ & 93.5 & 0.2 & & \pm 0.1 & 93.0 & 0.2 & 77.8 & \pm 0.2 & 97.2 & $=0.2$ & 89.5 \\
\hline JCDFA-M & 78.9 & $=0.3$ & 94.1 & 0.2 & & \pm 0.1 & 93.5 & 0.3 & 78.3 & \pm 0.1 & 97.3 & \pm 0.2 & 90.0 \\
\hline & $\mathrm{Ar} \rightarrow \mathrm{Cl}$ & $\mathrm{Ar} \rightarrow \mathrm{Pr}$ & $\mathrm{Ar} \rightarrow \mathrm{RW}$ & $\mathrm{Cl} \rightarrow \mathrm{Ar}$ & $\mathrm{Cl} \rightarrow \mathrm{Pr}$ & $\mathrm{Cl} \rightarrow \mathrm{Rw}$ & $\mathrm{Pr} \rightarrow \mathrm{Ar}$ & $\mathrm{Pr} \rightarrow \mathrm{Cl}$ & $\mathrm{Pr} \rightarrow \mathrm{RW}$ & $\mathrm{Rw} \rightarrow \mathrm{Ar}$ & $\mathrm{Rw} \rightarrow \mathrm{Cl}$ & $\mathrm{Rw} \rightarrow \mathrm{Pr}$ & Ave. \\
\hline JCDFA (w/o ada) & 53.2 & 71.3 & 75.9 & 62.4 & 71.2 & 69.4 & 61.6 & 51.1 & 75.8 & 68.4 & 51.3 & 79.8 & 66.0 \\
\hline JCDFA-C (w/o inter) & 50.3 & 74.5 & 79.4 & 63.6 & 71.6 & 73.7 & 64.5 & 49.5 & 80.6 & 68.9 & 53.3 & 80.2 & 67.5 \\
\hline JCDFA-C (w/o clu) & 58.2 & 78.6 & 81.5 & 69.3 & 75.2 & 76.7 & 67.3 & 55.3 & 82.0 & 73.0 & 60.0 & 82.6 & 71.6 \\
\hline JCDFA-C & 60.7 & 79.8 & 82.1 & 69.6 & 75.4 & 77.5 & 68.5 & 56.0 & 82.7 & 73.8 & 61.0 & 82.9 & 72.5 \\
\hline JCDFA-M (w/o clu) & 60.3 & 77.2 & 79.4 & 69.2 & 74.9 & 72.9 & 68.3 & 58.4 & 79.4 & 74.8 & 57.5 & 82.2 & 71.2 \\
\hline JCDFA-M & 61.7 & 78.1 & 83.2 & 69.8 & 77.9 & 78.6 & 69.8 & 59.7 & 83.4 & 75.9 & 63.4 & 85.9 & 74.0 \\
\hline
\end{tabular}

our JCDFA-C and JCDFA-M dramatically outperform all comparison methods on most tasks. The performance of JCDFA-M generally exceeds that of the other methods in terms of average accuracy, which is $74.0 \%$, representing a significant performance improvement of $2.7 \%$ compared to the best baseline SRDC. It is further noteworthy that JCDFA-C and JCDFA-M achieve significant improvement on the: $\mathrm{Cl} \rightarrow \mathrm{Ar}, \mathrm{Pr} \rightarrow \mathrm{Ar}$ and $\mathrm{Rw} \rightarrow \mathrm{Ar}$, which demonstrates the advantage of this method when dealing with the transfer from a complicated scenario to a simple scenario. Moreover, when encountering a large domain discrepancy, JCDFA still achieves strong performance on complex transfer tasks such as $\mathrm{Ar} \rightarrow \mathrm{Rw}, \mathrm{Cl} \rightarrow \mathrm{Rw}$ and $\mathrm{Pr} \rightarrow \mathrm{Rw}$, which further demonstrates the efficiency of the proposed JCDFA.

Results on VisDA-C VisDA-C is a more challenging dataset, as the objects in the target domain contain more variability and the domain shift between the two domains is much larger. The results in Table IV show that JCDFA-C achieves a more prominent improvement. In addition, JCDFA$\mathrm{C}$ performs much better than JCDFA-M, demonstrating that JCDFA-C has an advantage of dealing with complex transfer tasks in a large dataset.

\section{Analysis}

Ablation Studies We conducted ablation experiments on the Office-31, ImageCLEF-DA and Office-Home datasets to determine the effects of the different components in our JCDFA-C and JCDFA-M.

For JCDFA-C, we explored three components, i.e., the clustering, the discriminative features adaptation and the interclass contrastive learning. We eliminated the three components separately, where the training settings for these experiments are denoted as JCDFA-C (w/o clu), JCDFA-C (w/o ada), and JCDFA-C (w/o ada), respectively.

For JCDFA-M, we remove the clustering loss and the class-level adaptation loss from the overall training objective, respectively; the training settings for these experiments are denoted as JCDFA-M (w/o clu) and JCDFA-M (w/o ada), respectively. Notably, the JCDFA-C (w/o ada) and JCDFAM (w/o ada) denote the same model, so we use the JCDFA (w/o ada) to represent them for simplicity.

Table V presents the results of our ablation studies. JCDFAC significantly outperforms JCDFA-C (w/o inter), which demonstrates the inter-class contrastive learning plays an essential role in adapting the discriminative features cross domains. JCDFA-C (w/o clu) and JCDFA-M (w/o clu) perform much better than JCDFA (w/o ada), which shows that the adaptation of class-level features is more important than the clustering learning. JCDFA-C and JCDFA-M also outperform JCDFA-C (w/o clu) and JCDFA-M (w/o clu), respectively, which verifies that the mining of discriminative information via clustering learning plays an important role in this process. In addition, the ablation experiments reveal that the clustering learning and the adaptation of class-level features can promote each other and work better cooperatively.

Feature Visualization A popular method of visualizing high-dimensional data in 2D is t-SNE [60]. We visualize embedded features from ResNet-50, DANN, CDAN, our proposed JCDFA-C and JCDFA-M on the source and target domains for the adaptation task $\mathrm{A} \rightarrow \mathrm{W}$ and illustrate the results in Figure 5. The feature distributions of ResNet-50 can be seen from the figure to be disordered. DANN can alleviate the problem to a certain extent, however, there are still big discrepancies between the distributions of two domains. Although CDAN can improve the marginal distribution adaptation, mismatching of category-level features occurs. JCDFA$\mathrm{C}$ yields better class-level distribution alignment; however, compared to JCDFA-M, the marginal distribution of JCDFA$\mathrm{C}$ is somewhat more discrete, which is due to the lack of multi-layer features adaptation. JCDFA-M achieves the best adaptation results: specifically, the marginal and conditional distributions are well aligned, and the class boundary is clearly delineated.

Convergence Figure 6 (a)-(b) illustrate the test accuracy of several experimental methods on the $\mathrm{Ar} \rightarrow \mathrm{Cl}, \mathrm{Cl} \rightarrow \mathrm{Ar}, \mathrm{Pr} \rightarrow \mathrm{Ar}$ and $\mathrm{Ar} \rightarrow \mathrm{Rw}$ tasks, respectively. From 6 (a)-(b), we can see 


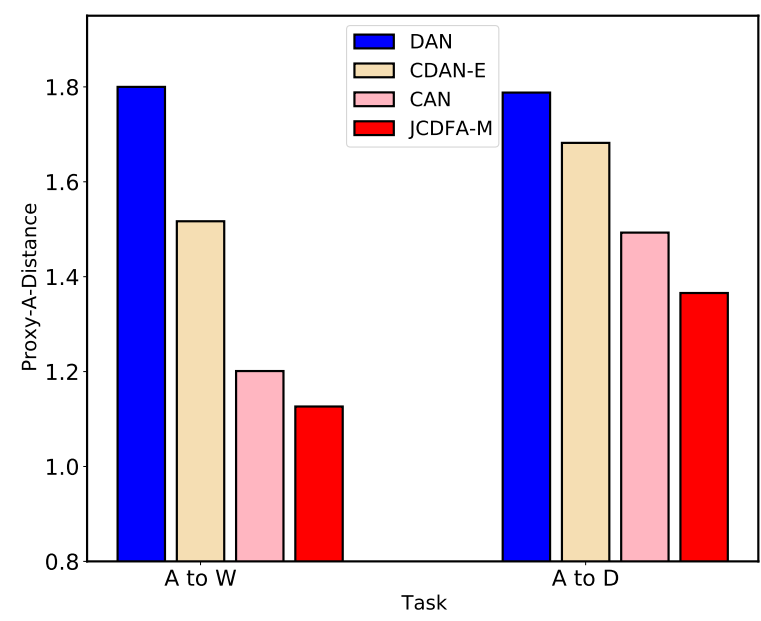

Fig. 7. Empirical analysis: Proxy $\mathcal{A}$-Distance of different features on $\mathrm{A} \rightarrow \mathrm{W}$ and $\mathrm{A} \rightarrow \mathrm{D}$.

JCDFA-C achieves optimal performance more quickly, which verifies the semi-supervised contrastive learning can adapt distributions effectively. There is an interesting finding that although JCDFA-C and JCDFA-M perform slightly better than with JCDFA-C (w/o clu) and JCDFA-M (w/o clu), respectively, JCDFA-C and JCDFA-M can achieve optimal performance much faster than JCDFA-C (w/o clu) and JCDFA$\mathrm{M}$ (w/o clu), which demonstrates that mining discriminative features via the clustering can accelerate the training process. Discrepancy Distance The theory of DA [71], [12] denotes the $\mathcal{A}$-distance as a measure of the cross-domain discrepancy, which will bound the target risk together with the source risk. The way to estimate the proxy $\mathcal{A}$-distance (PAD) is defined as $\hat{d}_{\mathcal{A}}=2(1-2 \epsilon)$, where $\epsilon$ is the generalization error of a binary classifier of discriminating source and target. We applied a kernel SVM to estimate the $\mathcal{A}$-distance. Figure 7 shows PADs on tasks $\mathrm{A} \rightarrow \mathrm{W}$ and $\mathrm{A} \rightarrow \mathrm{D}$ with features of DAN, CDAN+E, CAN and JCDFA-M. We observe that PAD of JCDFA-M is much smaller than the other comparison methods, which demonstrates that our features can reduce the cross-domain gap more effectively. The comparison with CAN shows that joint clustering learning and discriminative feature alignment has a greater advantage of learning domain-invariant discriminative features.

\section{CONCLUSION}

In this article, we develop a novel approach, i.e., JCDFA-C and JCDFA-M, for Unsupervised Domain Adaptation (UDA). The proposed JCDFA-C and JCDFA-M incorporate discriminative features learning and class-level features adaptation into a single framework. The discriminative features are achieved via joint learning of supervised classification of the source domain and unsupervised clustering of the target domain. The class-level feature adaptation procedure, which is based on the maximization of inter-class distances and the minimization of intra-class distances via an extended supervised contrastive learning or an extended MMD distance, can promote the transferability of discriminative features, as well as further benefit the class-wise distribution alignment across domains. These two procedures work cooperatively to improve the final target classification accuracy significantly. Comprehensive experiments on several UDA datasets clearly demonstrate that JCDFA substantially outperforms the stateof-the-art methods.

\section{REFERENCES}

[1] O. Russakovsky, J. Deng, H. Su, J. Krause, S. Satheesh, S. Ma, Z. Huang, A. Karpathy, A. Khosla, and M. Bernstein, "ImageNet large scale visual recognition challenge," IJCV, vol. 115, no. 3, pp. 211-252. 1

[2] L. Liu, W. Ouyang, X. Wang, P. Fieguth, J. Chen, X. Liu, and M. Pietikäinen, "Deep learning for generic object detection: A survey," $I J C V$, vol. 128 , no. 2, pp. 261-318, 2020. 1

[3] S. Minaee, Y. Boykov, F. Porikli, A. Plaza, N. Kehtarnavaz, and D. Terzopoulos, "Image segmentation using deep learning: A survey," arXiv preprint arXiv:1702.05374, 2020. 1

[4] A. Garcia-Garcia, S. Orts-Escolano, S. Oprea, V. Villena-Martinez, P. Martinez-Gonzalez, and J. Garcia-Rodriguez, "A survey on deep learning techniques for image and video semantic segmentation," Applied Soft Computing, vol. 70, pp. 41-65, 2018. 1

[5] Y. Taigman, M. Yang, M. Ranzato, and L. Wolf, "Deepface: Closing the gap to human-level performance in face verification," in CVPR, 2014, pp. $1701-1708.1$

[6] S. J. Pan, I. W. Tsang, J. T. Kwok, and Q. Yang, "Domain adaptation via transfer component analysis," in IJCAI, 2009, pp. 1187-1192. 1

[7] B. Gong, Y. Shi, F. Sha, and K. Grauman, "Geodesic flow kernel for unsupervised domain adaptation," in CVPR, 2012, pp. 2066-2073. 1

[8] I. Jhuo, D. Liu, D. T. Lee, and S. Chang, "Robust visual domain adaptation with low-rank reconstruction," in CVPR, 2012, pp. 21682175. 1

[9] H. Lu, C. Shen, Z. Cao, Y. Xiao, and A. van den Hengel, "An embarrassingly simple approach to visual domain adaptation," IEEE Trans. Image Process., vol. 27, no. 7, pp. 3403-3417, 2018. 1

[10] A. Torralba and A. A. Efros, "Unbiased look at dataset bias," in CVPR, 2011. 1

[11] S. Ben-David, J. Blitzer, K. Crammer, and F. Pereira, "Analysis of representations for domain adaptation," in NeurIPS, 2007, pp. 137-144. 1

[12] S. Ben-David, J. Blitzer, K. Crammer, A. Kulesza, F. Pereira, and J. W. Vaughan, "A theory of learning from different domains," Machine learning, vol. 79 , no. 1-2, pp. 151-175, 2010. 1, 12

[13] M. Wang and W. Deng, "Deep visual domain adaptation: A survey," Neurocomputing, vol. 312, pp. 135-153, 2018. 1

[14] G. Csurka, "Domain adaptation for visual applications: A comprehensive survey," arXiv preprint arXiv:1702.05374, 2017. 1

[15] W. M. Kouw and M. Loog, "A review of domain adaptation without target labels," TPAMI, 2020. 1

[16] Z. Lei, "Transfer adaptation learning: A decade survey," arXiv preprint arXiv:1903.04687, 2019. 1

[17] A. Chadha and Y. Andreopoulos, "Improved techniques for adversarial discriminative domain adaptation," IEEE Trans. Image Process., vol. 29, pp. 2622-2637, 2020. 1

[18] M. Long, Y. Cao, J. Wang, and M. I. Jordan, "Learning transferable features with deep adaptation networks," in ICML, 2015. 1, 3, 7, 9, 10

[19] M. Long, Y. Cao, Z. Cao, J. Wang, and M. I. Jordan, "Transferable representation learning with deep adaptation networks," TPAMI, 2018. 1

[20] K. Guoliang, J. Lu, Y. Yi, and H. Alexander, G, "Contrastive adaptation network for unsupervised domain adaptation," in CVPR, 2019, pp. 4893 4902. 1, 4, 7, 8, 9

[21] M. Long, H. Zhu, J. Wang, and M. I. Jordan, "Deep transfer learning with joint adaptation networks," in ICML, 2017, pp. 2208-2217. 1, 3, 9,10

[22] Y. Hongliang, D. Yukang, L. Peihua, W. Qilong, X. Yong, and Z. Wangmeng, "Mind the class weight bias: Weighted maximum mean discrepancy for unsupervised domain adaptation," in CVPR, 2017, pp. 2272-2281. 1, 3 
[23] Y. Hongliang, L. Zhetao, W. Qilong, L. Peihua, X. Yong, and Z. Wangmeng, "Weighted and class-specific maximum mean discrepancy for unsupervised domain adaptation," TMM, 2019. 1, 4

[24] B. Sun, J. Feng, and K. Saenko, "Return of frustratingly easy domain adaptation," in $A A A I, 2016.2,3$

[25] W. Zellinger, T. Grubinger, E. Lughofer, T. Natschläger, and S. Saminger-Platz, "Central moment discrepancy $(\mathrm{cmd})$ for domaininvariant representation learning," 2017. 2, 3

[26] Y. Ganin and V. Lempitsky, "Unsupervised domain adaptation by backpropagation," in ICML, 2014. 2, 3, 8

[27] Y. Ganin, E. Ustinova, H. Ajakan, P. Germain, H. Larochelle, F. Laviolette, M. Marchand, and V. Lempitsky, "Domain-adversarial training of neural networks," JMLR, vol. 17, no. 1, pp. 2096-2030, 2016. $2,3,8,9,10$

[28] E. Tzeng, J. Hoffman, K. Saenko, and T. Darrell, "Adversarial discriminative domain adaptation," in $C V P R, 2017$, pp. 7167-7176. 2, 3

[29] M. Long, Z. Cao, J. Wang, and M. I. Jordan, "Conditional adversarial domain adaptation," in NeurIPS, 2018, pp. 1640-1650. 2, 3, 8, 9, 10

[30] K. Saito, K. Watanabe, Y. Ushiku, and T. Harada, "Maximum classifier discrepancy for unsupervised domain adaptation," in CVPR, 2018, pp. 3723-3732. 2, 3, 9, 10

[31] C.-Y. Lee, T. Batra, M. H. Baig, and D. Ulbricht, "Sliced wasserstein discrepancy for unsupervised domain adaptation," in CVPR, 2019, pp. 10285-10295. 2, 3

[32] M. Ghifary, W. B. Kleijn, M. Zhang, D. Balduzzi, and W. Li, "Deep reconstruction-classification networks for unsupervised domain adaptation," in ECCV. Springer, 2016, pp. 597-613. 2

[33] J. Xie, R. B. Girshick, and A. Farhadi, "Unsupervised deep embedding for clustering analysis," in ICML, 2016, pp. 478-487. 2, 4, 6

[34] K. G. Dizaji, A. Herandi, C. Deng, W. Cai, and H. Huang, "Deep clustering via joint convolutional autoencoder embedding and relative entropy minimization," in ICCV, 2017, pp. 5747-5756. 2, 4, 5

[35] P. Khosla, P. Teterwak, C. Wang, A. Sarna, Y. Tian, P. Isola, A. Maschinot, C. Liu, and D. Krishnan, "Supervised contrastive learning," in Advances in Neural Information Processing Systems 33: Annual Conference on Neural Information Processing Systems 2020, NeurIPS 2020, December 6-12, 2020, virtual, 2020. 2, 4, 6

[36] G. Arthur, B. Karsten, R. Malte, S. Bernhard, and S. Alex, "A kernel two-sample test," JMLR, vol. 13, pp. 723-773, 2012. 2, 7

[37] B. Sun and K. Saenko, "Deep coral: Correlation alignment for deep domain adaptation," in ECCV, 2016, pp. 443-450. 3

[38] G. Ian, P.-A. Jean, M. Mehdi, X. Bing, W.-F. David, O. Sherjil, C. Aaron, and B. Yoshua, "Generative adversarial nets," in NeurIPS, 2014, p. 2672-2680. 3

[39] J. Shen, Y. Qu, W. Zhang, and Y. Yu, "Wasserstein distance guided representation learning for domain adaptation," in AAAI, 2018. 3

[40] C. Qingchao, L. Yang, W. Zhaowen, W. Ian, and C. Kevin, "Re-weighted adversarial adaptation network for unsupervised domain adaptation," in CVPR, 2018, pp. 7976-7985. 3

[41] Y. Zhang, H. Tang, K. Jia, and M. Tan, "Domain-symmetric networks for adversarial domain adaptation," pp. 5031-5040, 2019. 3, 9, 10

[42] H. Liu, M. Long, J. Wang, and M. Jordan, "Transferable adversarial training: A general approach to adapting deep classifiers," in ICML, 2019, pp. 4013-4022. 3, 9, 10

[43] X. Gu, J. Sun, and Z. Xu, "Spherical space domain adaptation with robust pseudo-label loss," in CVPR. IEEE, 2020, pp. 9098-9107. 3, 9, 10

[44] N. Xiao and L. Zhang, "Dynamic weighted learning for unsupervised domain adaptation," in CVPR, 2021, pp. 15 242-15 251. 3, 9

[45] Y. Shi and F. Sha, "Information-theoretical learning of discriminative clusters for unsupervised domain adaptation," in ICML, 2012. 3, 4

[46] Z. Deng, Y. Luo, and J. Zhu, "Cluster alignment with a teacher for unsupervised domain adaptation," in ICCV, 2019, pp. 9944-9953. 4, 9

[47] Y. Luo, J. Zhu, M. Li, Y. Ren, and B. Zhang, "Smooth neighbors on teacher graphs for semi-supervised learning," in CVPR, 2018, pp. 88968905. 4

[48] Y. Hsu, Z. Lv, and Z. Kira, "Learning to cluster in order to transfer across domains and tasks," in ICLR, 2018. 4

[49] H. Tang, K. Chen, and K. Jia, "Unsupervised domain adaptation via structurally regularized deep clustering," in CVPR, 2020, pp. 8722-8732. $4,8,9,10$

[50] L. Hu, M. Kan, S. Shan, and X. Chen, "Unsupervised domain adaptation with hierarchical gradient synchronization," in CVPR, 2020, pp. 40434052. $4,9,10$
[51] S. Xie, Z. Zheng, L. Chen, and C. Chen, "Learning semantic representations for unsupervised domain adaptation," in $I C M L, 2018$, pp. 5423-5432. 4, 9

[52] P. O. Pinheiro, "Unsupervised domain adaptation with similarity learning," in CVPR, 2018, p. 8004-8013. 4, 9, 10

[53] Y. Pan, T. Yao, Y. Li, Y. Wang, C. W. Ngo, and T. Mei, "Transferable prototypical networks for unsupervised domain adaptation," in $C V P R$, 2019, pp. 2239-2247. 4

[54] Z. Pei, Z. Cao, M. Long, and J. Wang, "Multi-adversarial domain adaptation," in AAAI, 2018. 4

[55] G. Chen, Y. Lu, J. Lu, and J. Zhou, "Deep credible metric learning for unsupervised domain adaptation person re-identification," in ECCV, ser. Lecture Notes in Computer Science, vol. 12353. Springer, 2020, pp. 643-659. 4

[56] A. Dosovitskiy, J. T. Springenberg, M. A. Riedmiller, and T. Brox, "Discriminative unsupervised feature learning with convolutional neural networks," in Advances in Neural Information Processing Systems, 2014, pp. 766-774. 4

[57] Z. Wu, Y. Xiong, S. X. Yu, and D. Lin, "Unsupervised feature learning via non-parametric instance discrimination," in CVPR, 2018, pp. 37333742. 4

[58] T. Chen, S. Kornblith, M. Norouzi, and G. E. Hinton, "A simple framework for contrastive learning of visual representations," in Proceedings of the 37th International Conference on Machine Learning, vol. 119. PMLR, 2020, pp. 1597-1607. 4

[59] Y. Ge, F. Zhu, D. Chen, R. Zhao, and H. Li, "Self-paced contrastive learning with hybrid memory for domain adaptive object re-id," in NeurIPS, 2020. 4

[60] V. D. M. Laurens and G. Hinton, "Visualizing data using t-sne," JMLR, vol. 9, pp. 2579-2605, 2008. 6, 11

[61] S. Li, C. H. Liu, L. Su, B. Xie, and D. Wu, "Discriminative transfer feature and label consistency for cross-domain image classification," TNNLS, 2020. 7

[62] S. Li, S. Song, G. Huang, Z. Ding, and C. Wu, "Domain invariant and class discriminative feature learning for visual domain adaptation," IEEE Trans. Image Process., vol. 27, no. 9, pp. 4260-4273, 2018. 7

[63] K. He, X. Zhang, S. Ren, and J. Sun, "Deep residual learning for image recognition," in CVPR, 2016, pp. 770-778. 8, 9, 10

[64] K. Saenko, B. Kulis, M. Fritz, and T. Darrell, "Adapting visual category models to new domains," in ECCV, 2010, pp. 213-226. 8

[65] H. Venkateswara, J. Eusebio, S. Chakraborty, and S. Panchanathan, "Deep hashing network for unsupervised domain adaptation," in CVPR, 2017, pp. 5018-5027. 8

[66] X. Peng, B. Usman, N. Kaushik, J. Hoffman, D. Wang, and K. Saenko, "Visda: The visual domain adaptation challenge," arXiv preprint arXiv:1710.06924, 2017. 8

[67] T. Y. Lin, M. Maire, S. Belongie, L. Bourdev, R. Girshick, J. Hays, P. Perona, D. Ramanan, C. L. Zitnick, and P. Dollár, "Microsoft coco: Common objects in context," in ECCV, 2014, pp. 740-755. 8

[68] J. Deng, W. Dong, R. Socher, L. Li, K. Li, and F. Li, "Imagenet: A large-scale hierarchical image database," in CVPR, 2009, pp. 248-255. 8

[69] P. Adam, G. Sam, C. Soumith, C. Gregory, Y. Edward, D. Zachary, L. Zeming, D. Alban, A. Luca, and L. Adam, "Automatic differentiation in pytorch," JMLR, 2017. 8

[70] K. You, X. Wang, M. Long, and M. I. Jordan, "Towards accurate model selection in deep unsupervised domain adaptation," in ICML, vol. 97. PMLR, 2019, pp. 7124-7133. 8

[71] Y. Mansour, M. Mohri, and A. Rostamizadeh, "Domain adaptation: Learning bounds and algorithms," in COLT, 2009. 12 


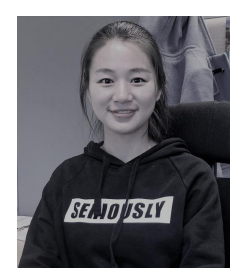

Wanxia Deng received the university B.E. degree in electronic information science and technology from Xiamen University, Xiamen, China, and the M.S. degree in information and communication engineering from the National University of Defense Technology (NUDT), Changsha, China, in 2014 and 2016, respectively. She is currently pursuing the Ph.D. degree in information and communication engineering from the National University of Defense Technology, Changsha, China. Her research interests include domain adaptation, transfer learning, deep learning and image processing.

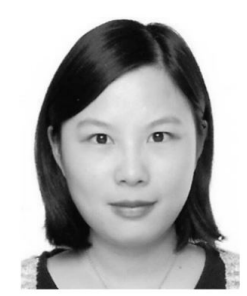

Qing Liao received B.Sc. degree in software technology and application from Macau University of Science and Technology, Macau, in 2010, the M.Phil. degree in computer science and technology from the Fok Ying Tung Graduate School of Hong Kong University of Science and Technology in 2013 and the Ph.D. degree from the Department of Computer Science and Engineering, Hong Kong University of Science and Technology, in 2016. She is currently an Assistant Professor with the School of Computer Science and Technology, Harbin Institute of Technology (Shenzhen), China. Her research interests include artificial intelligence and bioinformatics.

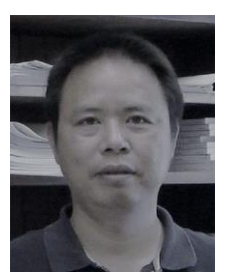

Gangyao Kuang received the B.S. and M.S. degrees from the Central South University of Technology, Changsha, China, in 1988 and 1991, respectively, and the Ph.D. degree from the National University of Defense Technology, Changsha, China, in 1995. He is currently a Professor at School of Electronic Science, National University of Defense Technology. He was a Visiting Scholar with the University of Waterloo, Canada, from 2009 to 2010. His current interests include remote sensing, SAR image processing, change detection, SAR ground moving target indication, and the classification of polarimetric SAR images.

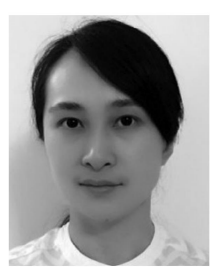

Lingiun Zhao received the B.S., M.S., and Ph.D. degrees in information and communication engineering from National University of Defense Technology, Changsha, China, in 2003, 2004, and 2009 , respectively. She is currently an associate professor with the School of Electronic Science, National University of Defense Technology. Her research interest covers remote sensing information processing, SAR automatic target recognition and deep learning.

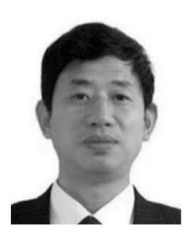

Dewen Hu received the B.S. and M.S. degrees from Xi'an Jiaotong University, China, in 1983 and 1986, respectively, and the Ph.D. degree from the National University of Defense Technology in 1999. He is currently a Professor at School of Intelligent Science, National University of Defense Technology. From October 1995 to October 1996, he was a Visiting Scholar with the University of Sheffield, U.K. His research interests include image processing, system identification and control, neural networks, and cognitive science.

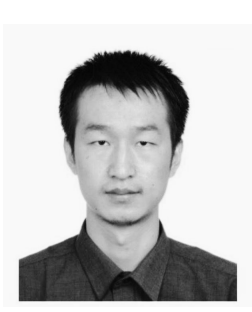

Deke Guo received the B.S. degree in industry engineering from the Beijing University of Aeronautics and Astronautics, Beijing, China, in 2001, and the Ph.D. degree in management science and engineering from the National University of Defense Technology, Changsha, China, in 2008. $\mathrm{He}$ is currently a Professor with the College of System Engineering, National University of Defense Technology, and is also with the College of Intelligence and Computing, Tianjin University. His research interests include distributed systems, software-defined networking, data center networking, wireless and mobile systems, and interconnection networks.

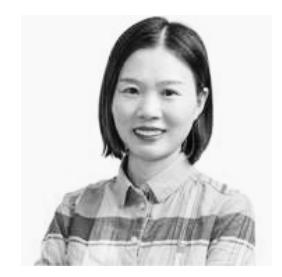

$\mathrm{Li}$ Liu received the $\mathrm{Ph} . \mathrm{D}$. degree in information and communication engineering from the National University of Defense Technology (NUDT), China, in 2012. She is currently a Professor with the College of System Engineering. During her PhD study, she spent more than two years as a Visiting Student at the University of Waterloo, Canada, from 2008 to 2010. From 2015 to 2016, she spent ten months visiting the Multimedia Laboratory at the Chinese University of Hong Kong. From 2016.12 to 2018.11, she worked as a senior researcher at the Machine Vision Group at the University of Oulu, Finland. Her current research interests include computer vision, pattern recognition and machine learning. Her papers have currently over 3700 citations in Google Scholar. 